\title{
Energy Dissipation Mechanism and Control Model of a Digital Hydraulic Damper
}

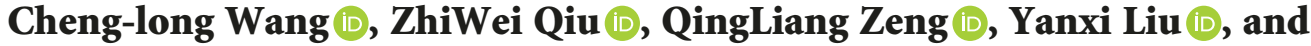 \\ Genyuan Miao \\ Shandong University of Science and Technology, College of Mechanical and Electronic Engineering, 266590 Qingdao, China \\ Correspondence should be addressed to QingLiang Zeng; qlzeng@sdust.edu.cn
}

Received 19 April 2019; Revised 4 August 2019; Accepted 16 August 2019; Published 13 October 2019

Guest Editor: Efrén Díez-Jiménez

Copyright (c) 2019 Cheng-long Wang et al. This is an open access article distributed under the Creative Commons Attribution License, which permits unrestricted use, distribution, and reproduction in any medium, provided the original work is properly cited.

\begin{abstract}
In order to achieve the high efficiency and stable cushioning effect under the uncertain impacting situation, a digital hydraulic damper is developed which can adjust the whole buffering capacity so as to be adaptable to the impacting load on time during the buffering process. In the paper, the mechanism of energy dissipation in the digital hydraulic damper and the whole damping process are studied. The relationship between the energy dissipation process and the control process is given. Firstly, the energy dissipation process of the digital throttling unit is studied, and the mathematical model of energy losses of the digital hydraulic damper unit and the energy dissipation model of the whole digital damper are established. The energy dissipation model of the digital hydraulic buffer is proposed. Secondly, the effect on energy losses caused by the three elements of the digital damper, which are the sudden extension of cross section, form of orifice, and form of cone valve port, is analyzed. Finally, based on the energy dissipation model, the control model of the energy dissipation process is given, which lays a theoretical foundation for detailed structural design and control optimization.
\end{abstract}

\section{State of the Art}

As a kind of safety protection device, the hydraulic damper has been widely used in various occasions. The basic working principle is that the energy generated by the impacting body is consumed by means of throttle section which can produce liquid resistance.

Jiang [1] studied the gradual throttling hydraulic buffer, and the role of gradient throttle hydraulic buffer in a tracked vehicle suspension system is analyzed. Wang et al. [2] studied the structure design, optimization, and dynamic characteristics of multiorifice hydraulic buffer. Wang and Shen [3] simulated and analyzed the viscoelastic damper; an optimum method of clay buffer design was proposed. Qi [4] made a comparative study of the experiment and simulation of the viscoelastic buffer, which indicates high feasibility of the application of the viscoelastic material in the recoil mechanism. Wang et al. [5] studied the cushioning characteristics of viscoelastic buffer, which shows that the use of viscoelastic damper can increase the energy absorption rate and improve the stability. Baochen et al. [6] studied and analyzed the buffer characteristics of throttle bar buffer and verified the rationality of the selection method of $K$.

For the buffer, the prompt response and the smooth buffering process should be improved on the basis of guaranteeing the buffer efficiency and amount of absorbed shocking energy. In order to realize the above goals, a digital hydraulic buffer is proposed by Wang et al. [7]. In the buffer as shown in Figure 1, the throttle valve and high-speed on-off valve are connected in series to form a digital magnetic throttle unit, and the digital throttle valve group is composed of several groups of digital magnetic throttle units in parallel; high-speed on-off valve controlled by PID is used to complete the variable throttle control.

For the digital hydraulic buffer, in order to achieve the high efficiency and stable cushioning effect under the uncertain impact load, the following problems should be solved: firstly, for the digital throttling unit, the key point 


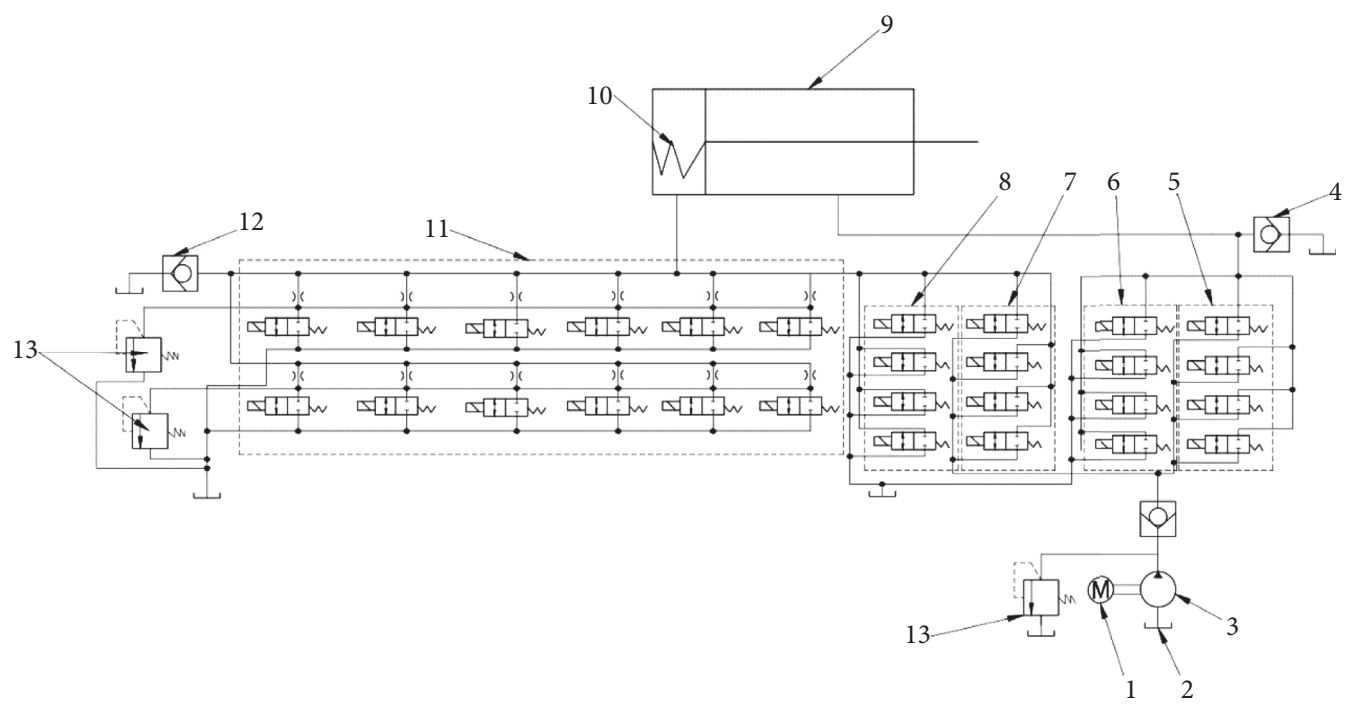

Figure 1: Digital buffer hydraulic system. (1) Motor; (2) tank; (3) gear pump; (4) check valve for rod chamber; (5) group of oil inlet control valves for rod chamber; (6) group of oil outlet control valves for the rod chamber; (7) group of oil inlet control valves for the rodless chamber; (8) group of oil outlet control valves for the rodless chamber; (9) cylinder; (10) reset spring; (11) digital throttle unit; (12) check valve for the rodless chamber; (13) safety valve.

is how to ensure the appropraite PWM (pulse width modulation) and value of energy dissipation during the buffering process, and secondly, how to control the whole buffering capacity so as to make it adaptable to the impacting load on time during the buffering process.

In order to achieve the above objectives, the energy dissipation process of the digital throttling unit needs to be studied. On this basis, the energy dissipation model and the control model of the digital hydraulic buffer should be proposed, which will provide a theoretical foundation for detailed structural design and control optimization.

Aiming at the problem of energy dissipation, Hong et al. [8-10] studied the flow coefficient and flow field variation law of various valve ports. Zhang et al. [11] have carried out the experimental research on the flow coefficient of the orifice of flat plate valve, conical valve, ball valve, and slide valve and put forward the optimum design for the structural design of various orifices from the flow coefficient point of view. $\mathrm{Wu}[12,13]$ analyzed the flow characteristics of servo valve in the laminar and turbulent state by means of experiment. Srikanth and Bhasker [14] studied the formation and development of vortices in the flow field of hydraulic valves based on the dynamic grid technology. Pace et al. [15] studied the flow coefficient of the spool valve orifice and the liquid change of the internal flow field. Liu et al. [16] studied the wake flow field of cone flow meter. Yu et al. [17] studied the effect of orifice chamfer on the flow field characteristics of perforated plate flow meter. Gao [18] carried out finite element analysis on the flow field of the complex flow passage and various throttle holes. Wang et al. [19] studied the causes of cavitations' generation and influencing factors of the hydraulic poppet valve port. $\mathrm{Fu}$ and $\mathrm{Du}[20]$ studied the cavitations' phenomenon in high-speed flow of throttle groove. Vallet et al. [21] studied the hydraulic pressure of the hydraulic spool and the flow coefficient of the valve orifice.

Among the above research, the mechanism of energy losses in the whole damping system and the whole damping process has not been systematically studied. The relationship between the energy dissipation process and the control process is not mentioned. In our research, the energy dissipation process of the digital throttling unit is studied, and the influence of the sudden extension of digital damper unit, valve opening, and extension on energy losses is analyzed. Energy dissipation model of the digital hydraulic buffer is proposed, and the correlative model of energy dissipation and control processes is established, which lays a theoretical foundation for detailed structural design and control optimization.

\section{Energy Dissipation Mechanism of the Digital Hydraulic Damper}

A digital hydraulic damper consists of several digital magnetic throttle units which are made up of the high-speed on-off valve and the throttle valve. The throttle valve and the high-speed on-off valve are connected in series so as to form the digital throttling unit shown in Figure 2, which consists of the throttle valve body, coil, valve core, gas spring, keeper, and pole shoe. A number of digital magnetic throttle units are combined together in parallel to form a digital throttle valve group. When the oil passes through the unit, there are local pressure losses and frictional pressure losses, and a damping force is created so as to buffer the impacting object until it stops.

As shown in Figure 3, when the fluid flows through the digital throttling unit, there are three kinds of energy losses which are local pressure losses at sudden change cross 


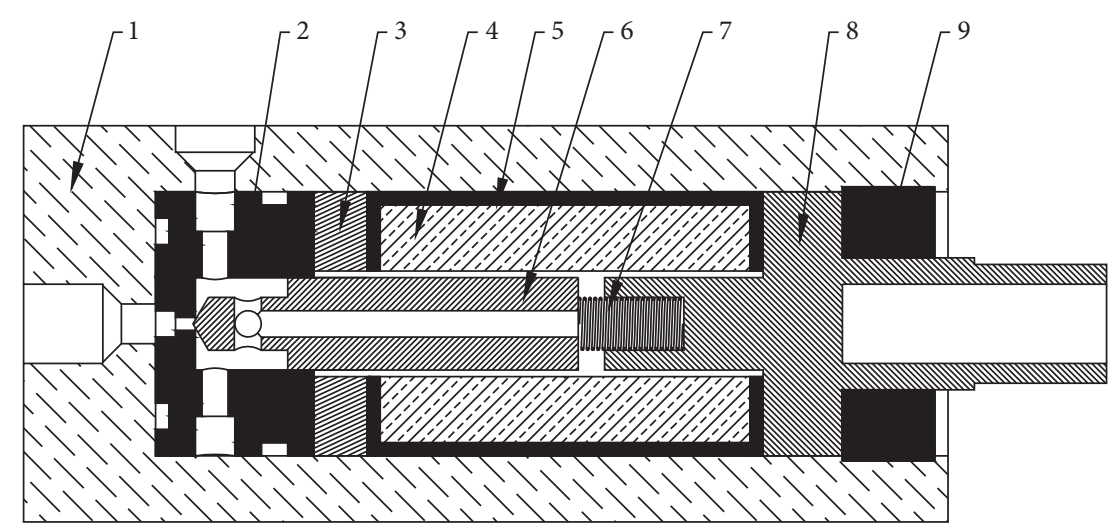

FIGURE 2: Digital magnetic throttle unit. (1) Integrated valve block; (2) valve body; (3) ring iron; (4) coil; (5) yoke; (6) spool; (7) reset spring; (8) iron core; (9) fastening ring.

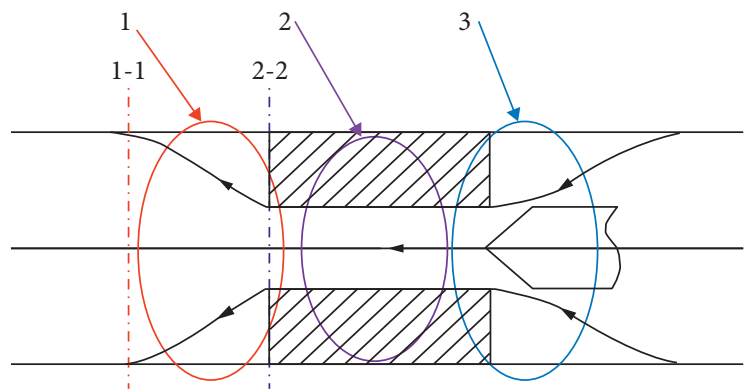

FIGURE 3: Energy losses of digital damping unit. (1) Local pressure losses at sudden change cross section; (2) friction pressure losses through the orifice; (3) local pressure losses through the cone valve port.

section, frictional pressure losses through the orifice, and local pressure losses through the cone valve port. A mathematical model for the energy losses of the unit is analyzed according to the above three factors.

\subsection{Mathematical Model for the Energy Losses of the Unit.} The flow rate of the digital damper unit depends on the flow capacity of the conical valve orifice. In a pulse width modulation (PWM) period, the average flow rate through the valve orifice is proportional to the PWM of the input signal. The average flow rate of the digital damper unit is as follows:

$$
\bar{q}=\tau C_{\mathrm{d}} A_{2} \sqrt{\frac{2 \Delta p_{2}}{\rho}} .
$$

In the formula, $\bar{q}$ is the average flow rate of the digital damper unit, $\tau$ is the PWM of pulse, which is equal to $t_{\mathrm{p}} / t_{\mathrm{f}}$, where $t_{\mathrm{p}}$ is the pulse width of PWM and $t_{\mathrm{f}}$ is the pulse period of PWM, $C_{\mathrm{d}}$ is the flow coefficient, $A_{2}$ is the area of throttle hole at section 2-2, and $\Delta p_{2}$ is the pressure difference of the conical valve orifice.

2.1.1. Local Pressure Losses at Sudden Change Cross Section. In Figure 3, local pressure losses at sudden change cross section are illustrated when the fluid flows into the orifice of the digital magnetic throttle unit. $h_{\mathrm{m}}$ (local pressure losses at sudden change cross section) can be described as follows:

$$
h_{\mathrm{m}}=\left(1-\frac{A_{2}}{A_{1}}\right)^{2} \frac{v_{2}^{2}}{2 g}=\zeta \frac{v_{2}^{2}}{2 g} .
$$

In formula (2), $A_{2}$ is the area of section 2-2, $A_{1}$ is the area of section $1-1, v_{2}$ is the average velocity of flow in section $2-2$, $\zeta$ is the partial loss coefficient which is equal to $1-\left(A_{2} / A_{1}\right)^{2}$ and $g$ is the gravity acceleration. According to formula (2),

$$
v_{2}=\frac{\bar{q}}{A_{2}}=\frac{\tau C_{\mathrm{d}} A_{2}}{A_{2}} \sqrt{\frac{2 \Delta p_{2}}{\rho}} .
$$

The energy losses at cross section changes are as follows:

$$
h_{\mathrm{m}}=\frac{\zeta \Delta p_{2}}{\rho g}\left(\frac{\tau C_{\mathrm{d}}}{1}\right)^{2} .
$$

2.1.2. Frictional Pressure Losses through the Orifice. According to the Navier-Stokes equation, the pressure losses $\Delta p_{1}$ flow through the distance of 1 are as follows:

$$
\Delta p_{1}=\frac{32 \mu l v_{2}}{d^{2}}
$$

In formula (5), $\mu$ is the kinematic viscosity of the fluid, $d$ is the diameter of the throttle hole, and $v_{2}$ is the velocity of the fluid in the throttle hole. According to formulas (3) and (5), it shows that the pressure losses are as follows: 


$$
\Delta p_{1}=\frac{32 \mu l \tau C_{\mathrm{d}}}{d^{2}} \sqrt{\frac{2 \Delta p_{2}}{\rho}} .
$$

2.1.3. Local Pressure Losses through the Cone Valve Port. The buffering process is realized by means of a number of parallel digital damper units. If the number of opened digital magnetic throttle units is $n$ at the moment, then the average flow $\bar{q}$, which flows through the port of the cone spool, is as follows:

$$
\bar{q}=\frac{A v}{n} .
$$

In formula (7), $A$ is the cross-sectional area of the digital hydraulic damper piston which is shown in Figure 1, and $v$ is the motion velocity of the piston at this moment. Pressure losses caused by the cone spool of the digital magnetic throttle unit are as follows:

$$
\Delta p_{2}=\frac{\rho}{2}\left(\frac{A v}{n \tau C_{\mathrm{q}} A_{2}}\right)^{2} .
$$

2.1.4. Energy Dissipation Model of the Digital Hydraulic Damper. In summary, total losses of each digital damping unit in the digital hydraulic damper include three parts which are losses in the sudden extension section, losses along throttle orifice, and local losses at cone valve orifice. The mathematical model of total losses per digital damping unit (water column height) can be described as follows:

$$
\begin{aligned}
h= & \frac{\Delta p_{1}}{\rho}+\frac{\Delta p_{2}}{\rho}+h_{\mathrm{m}}=\frac{32 \mu l \tau C_{\mathrm{d}}}{\rho d^{2}} \sqrt{\frac{2 \Delta p_{2}}{\rho}} \\
& +\frac{1}{2}\left(\frac{A v}{n \tau C_{\mathrm{q}} A_{2}}\right)+\frac{\zeta \Delta p_{2}}{\rho g}\left(\frac{\tau C_{\mathrm{d}}}{1}\right)^{2} .
\end{aligned}
$$

Here, the energy dissipation model of the whole system is given as follows:

$$
H=\sum h i, \quad i=1,2,3, \ldots, n .
$$

2.2. Simulation Analysis of Energy Losses of Digital Damping Unit. In this section, the three factors affecting the energy losses of the digital damping unit are mainly studied, which are, namely, the sudden extension channel with chamfer, sections with different proportions of sudden extension, and conical spool with different angles. Focusing on the three factors, the simulation analysis is done.

The flow field modeling of the channel with sudden extension can be established according to Figure 4 and Table 1. The flow channel of the digital damping unit is shown in Figure 4, which has a damping hole. The diameter of the hole is $0.5 \mathrm{~mm}$ and the length of the hole is $0.75 \mathrm{~mm}$.

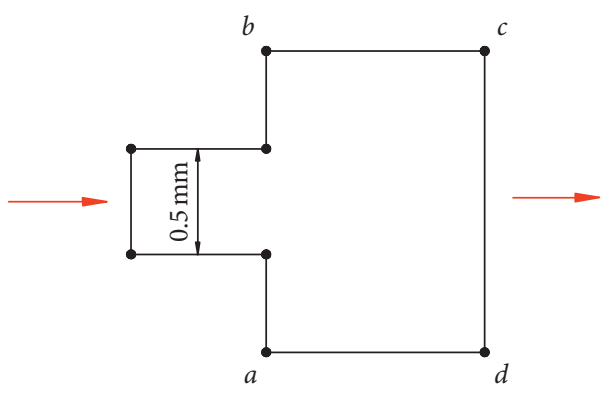

FIgURE 4: Structure of channel with sudden extension.

The size of sections with different proportions of sudden extension is obtained according to Table 1.

Here, 45\# hydraulic oil is used in the simulation and the specific simulation parameters are shown in Table 2.

The flow field modeling of the conical valve core is established according to Figures 5 and 6 and Table 3 . The conical valve core in Figures 5 and 6 has the same opening height. The height is $0.3 \mathrm{~mm}$. The size of the conical spool with different angles is obtained according to Table 3 .

\subsection{Influence of Extension of Cross Section with Different} Ratios. After the fluid flows through the sudden extension of cross section, the flow field diagram shown in Figure 7 is formed. It can be seen that the jet flow phenomenon occurs in the cross section with different sudden extension proportions, resulting in the existence of the wall recirculation zone and the jet recirculation zone. The key factor of energy losses is swirls of various changes in the recirculation zone. Since the recirculation zone between the jets is small, it can be neglected. Here, $L$ is the length of the recirculation zone and $v$ is the flow velocity of the recirculation zone. The starting point of the sudden extension of cross section is taken as the coordinate origin, and coordinate map of the flow field of the section is established. The coordinates of vortex center of the recirculation zone of the upper and lower walls are represented by $\mathrm{O}_{1}$ and $\mathrm{O}_{2}$.

From Figure 8 and Table 4 , it can be seen that the pressure is changed seriously when the sudden spread ratio is equal to 1.5. From Figures 9-15, it can also be seen that with the increase of the sudden spread ratio, scale of the vortex and the negative pressure region is increased. However, from Figure 16 and 17, it can be seen that the sudden spread ratio is inversely proportional to the turbulent kinetic energy and velocity of the vortex center reflux. In summary, the scale of the vortex is not the key factor of energy losses. The key factors which determine the energy losses in the sudden spread of the cross section are the reflux speed and turbulent kinetic energy in the reflux zone. The smaller the sudden spread ratio, the greater the confluence velocity and turbulent kinetic energy in the reflux zone. And the more complex the 
TABLE 1: Structure size of sections with different proportions of sudden extension.

\begin{tabular}{|c|c|c|c|c|}
\hline \multirow{2}{*}{$\begin{array}{l}\text { Name } \\
\text { Cross sections with proportion of sudden extension }\end{array}$} & \multicolumn{4}{|c|}{ Coordinate (unit: $\mathrm{mm}$ ) } \\
\hline & $a$ & $b$ & $c$ & $d$ \\
\hline 1.25 & $(0,0)$ & $(0.625,0)$ & $(0.625,0.75)$ & $(0,0.75)$ \\
\hline 1.5 & $(0,0)$ & $(0.75,0)$ & $(0.75,0.75)$ & $(0,0.75)$ \\
\hline 1.75 & $(0,0)$ & $(0.875,0)$ & $(0.875,0.75)$ & $(0,0.75)$ \\
\hline 2 & $(0,0)$ & $(1,0)$ & $(1,0.75)$ & $(0,0.75)$ \\
\hline
\end{tabular}

TABLE 2: Simulation parameters.

\begin{tabular}{lccc}
\hline Model & Viscosity $(\mathrm{kg} / \mathrm{m} \cdot \mathrm{s})$ & Density $\left(\mathrm{kg} / \mathrm{m}^{3}\right)$ & Inlet velocity $(\mathrm{m} / \mathrm{s})$ \\
\hline 45\#hydraulic oil & 0.04 & 890 & 15
\end{tabular}

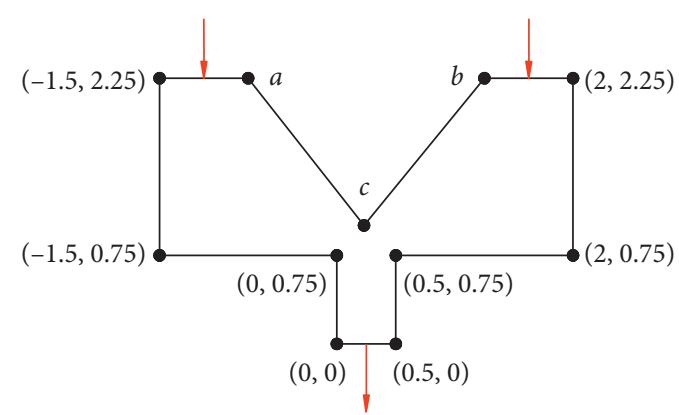

Figure 5: Structural sketch of $60^{\circ}$ conical valve core.

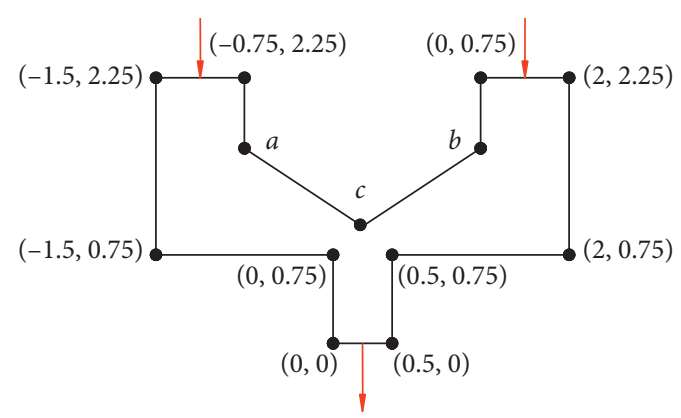

FIgURE 6: Structural sketch of $90^{\circ}$ and $120^{\circ}$ conical valve core.

TABLE 3: Structural size of the conical spool with different angles.

\begin{tabular}{lccc}
\hline $\begin{array}{l}\text { Name } \\
\text { Angle }\end{array}$ & $a$ & Coordinate (unit: mm) & $c$ \\
\hline 60 & $(-0.807,2.25)$ & $(1.193,2.25)$ & $(0.25,0.617)$ \\
90 & $(-0.75,1.05)$ & $(0.75,1.05)$ & $(0.25,0.8)$ \\
120 & $(-0.75,0.733)$ & $(0.75,0.733)$ & $(0.25,0.906)$ \\
\hline
\end{tabular}

structure of the vortex in the reflux zone, the more intense the motion and the greater the local pressure losses. From Figure 18, it can also be verified that the pressure difference between inlet and outlet is inversely proportional to the sudden spread ratio, and the flow coefficient is proportional to the sudden spread ratio. It shows that the larger the sudden spread ratio, the smaller the energy losses.

2.4. Chamfer Effect on Energy Losses of Sudden Extension of Cross Section. From Figures 19 and 20 and Table 5, it can 


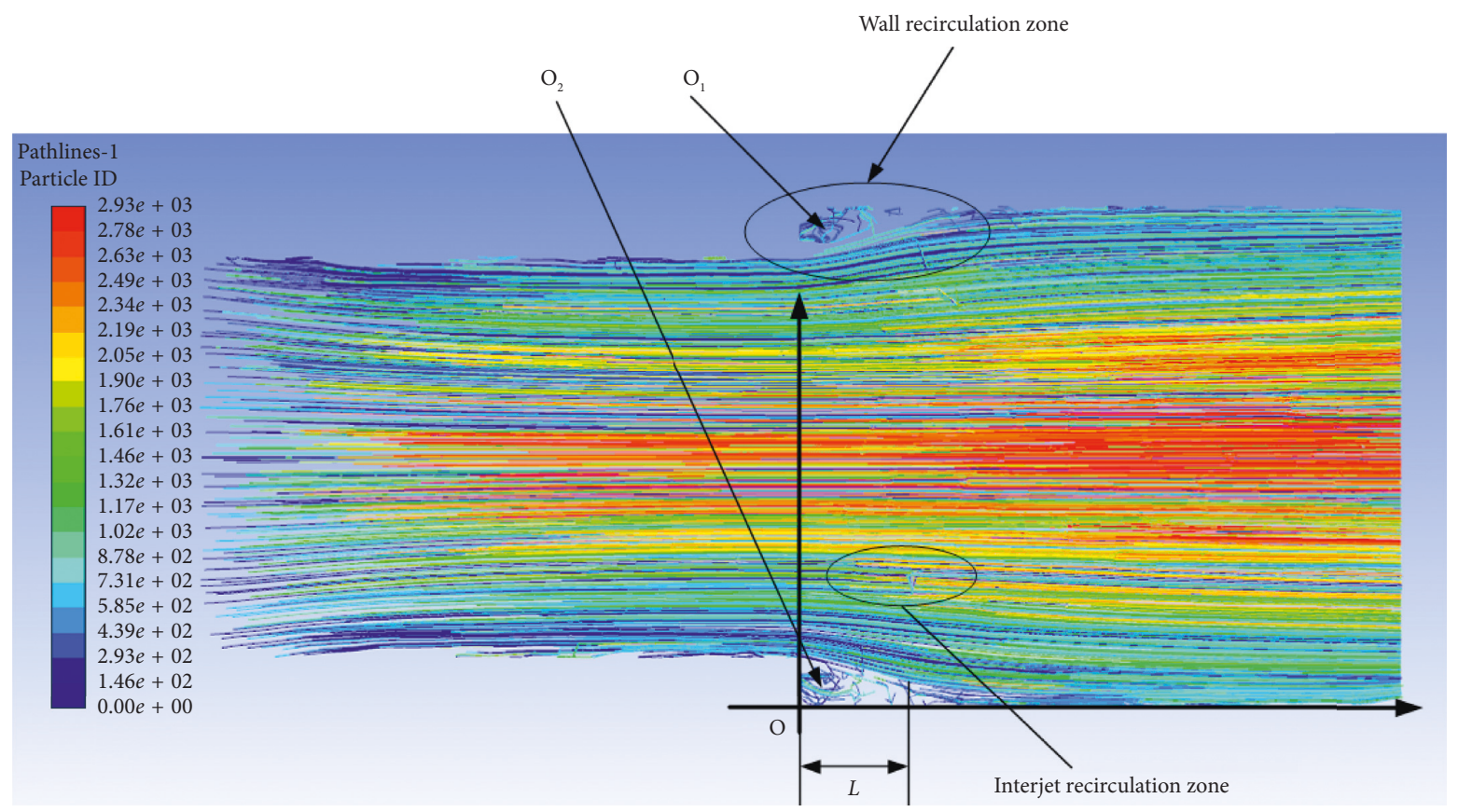

Figure 7: Flow field diagram of the sudden extension of cross section.

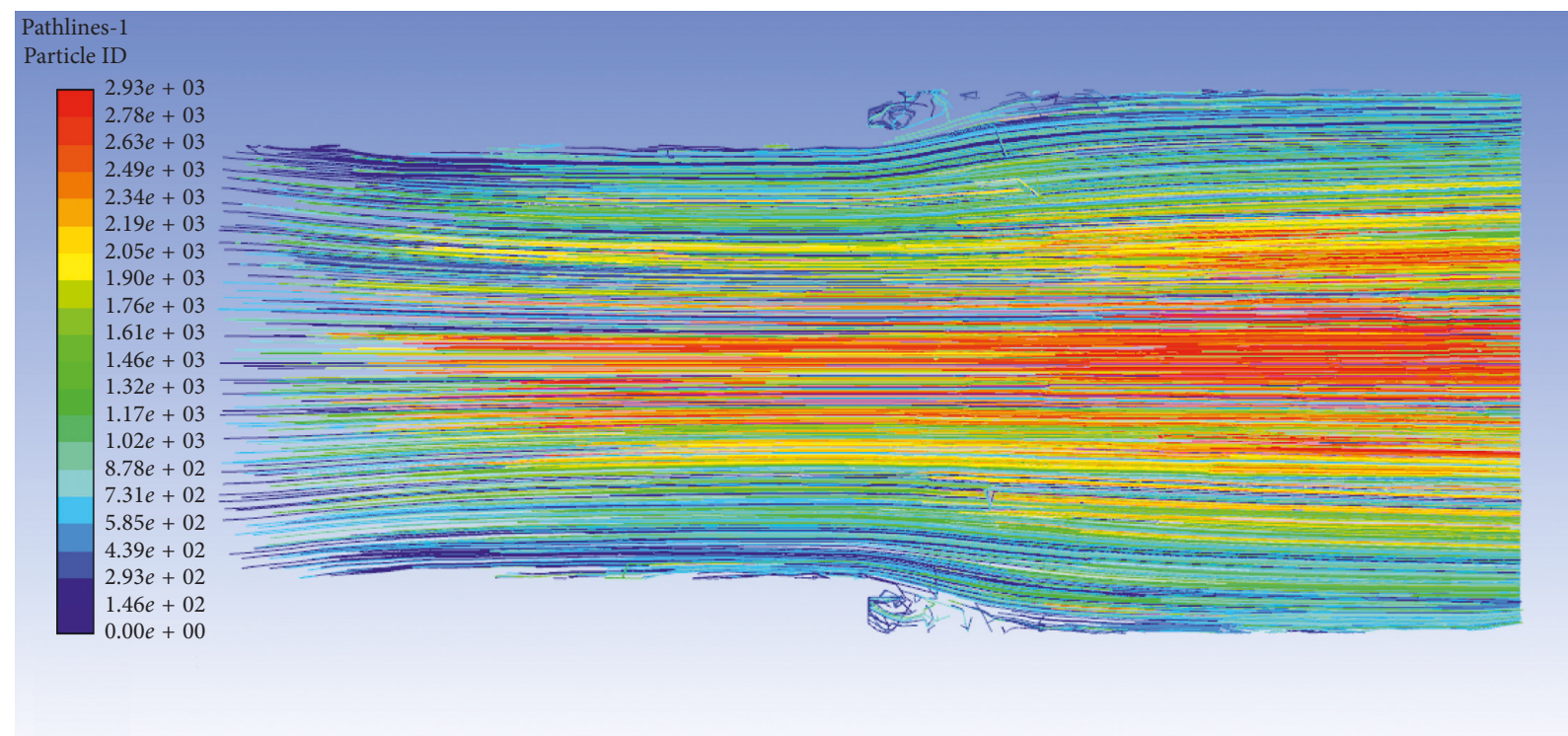

Figure 8: Sudden extension ratio is 1.25 .

TABLE 4: Characteristics of sudden extension reflux zone in cross section.

\begin{tabular}{lccc}
\hline Inlet condition & Vortex position & Vortex structure & Reflow zone length \\
\hline & $\mathrm{O}_{1}(0.067 D, 0.067 D)$ & Complex structure and intense movement & $0.33 D$ \\
$15 \mathrm{~m} / \mathrm{s}$ & $\mathrm{O}_{1}(0.18 D, 0.16 D)$ & Stable reattachment vortex & $0.94 D$ \\
& $\mathrm{O}_{1}(0.4 D, 0.33 D)$ & Stable reattachment vortex & $1.33 D$ \\
& $\mathrm{O}_{1}(0.62 D, 0.31 D)$ & Stable reattachment vortex & $1.54 D$ \\
\hline
\end{tabular}

$D$ is the diameter of the damping hole. 


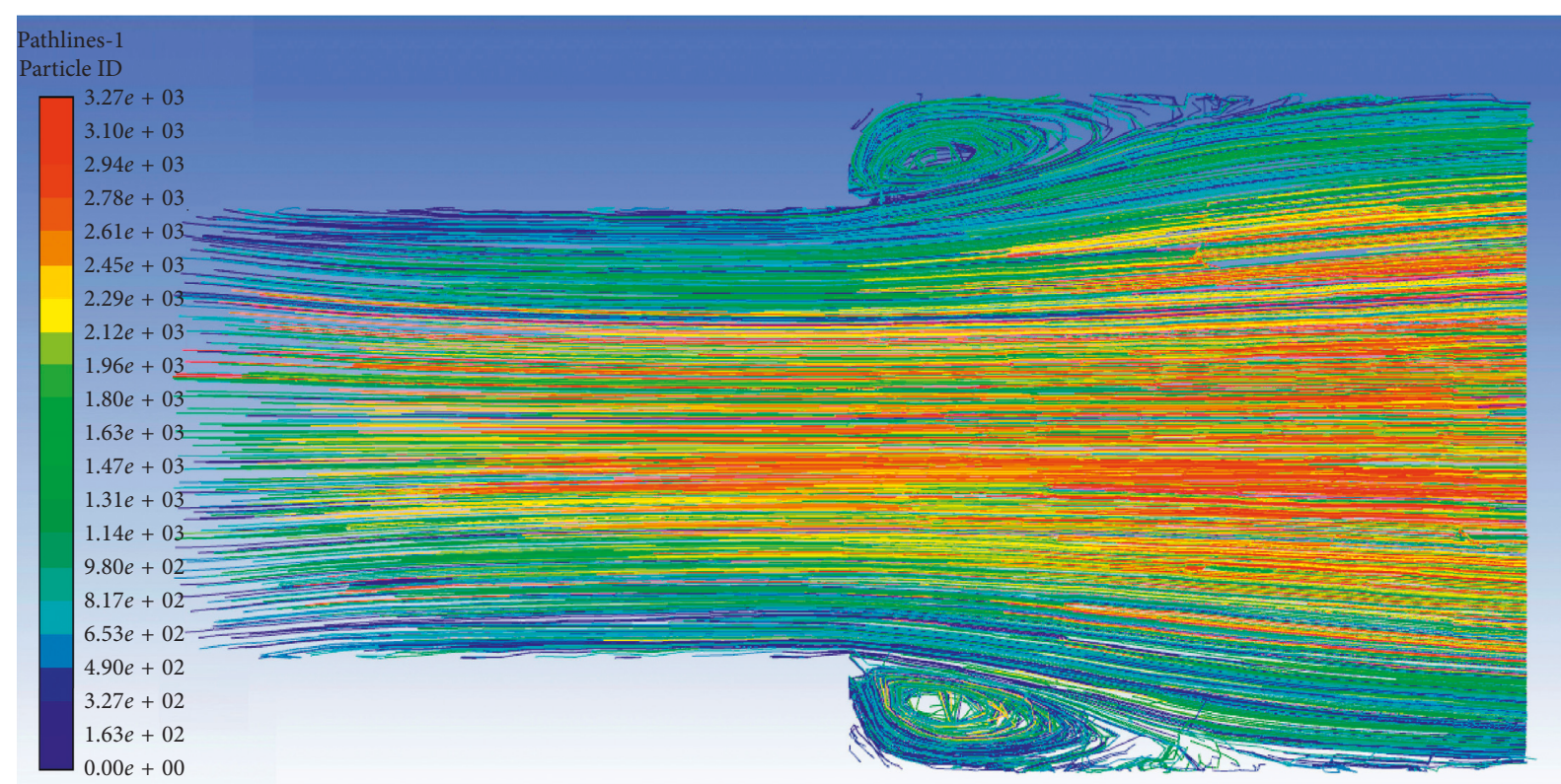

Figure 9: Sudden extension ratio is 1.5.

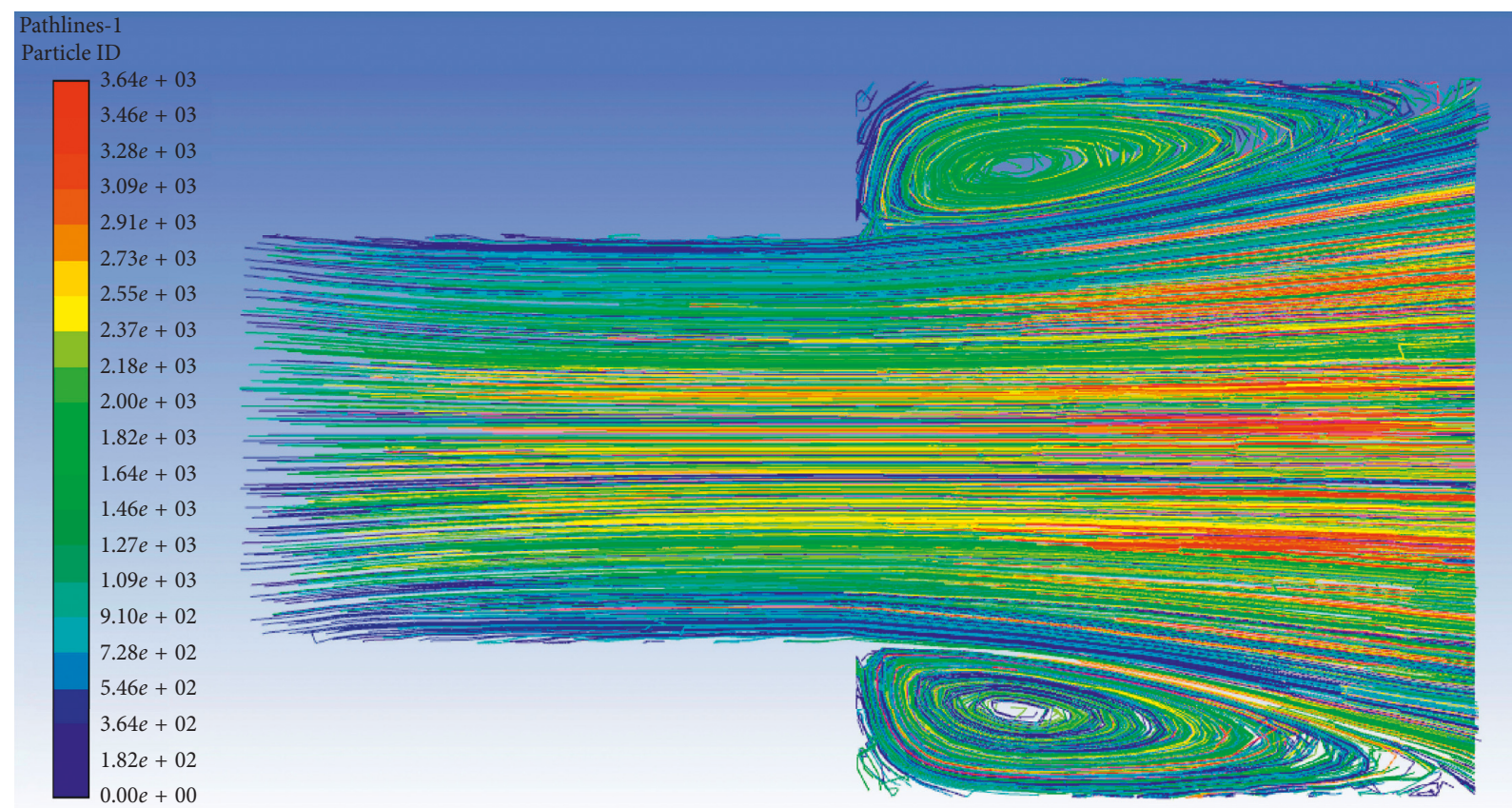

FIgURE 10: Sudden extension ratio is 1.75 .

be seen that if there is a chamfer at extension of cross section, the size of the vortex at the extension of cross section will be reduced, and the negative pressure zone will also be reduced. From Figures 21 and 22, the same result can be obtained. From Figure 23, it can be seen that the turbulent kinetic energy of extension of cross section with chamfer is lower than that of the sudden extension of cross section without chamfer. From Figure 24, it can be seen that the reflux velocity of vortex center of the sudden extension of cross section with chamfer is lower than that of the sudden extension of cross section without chamfer.

The pressure losses at the sudden extension of cross section are mainly due to the fact that the streamline 


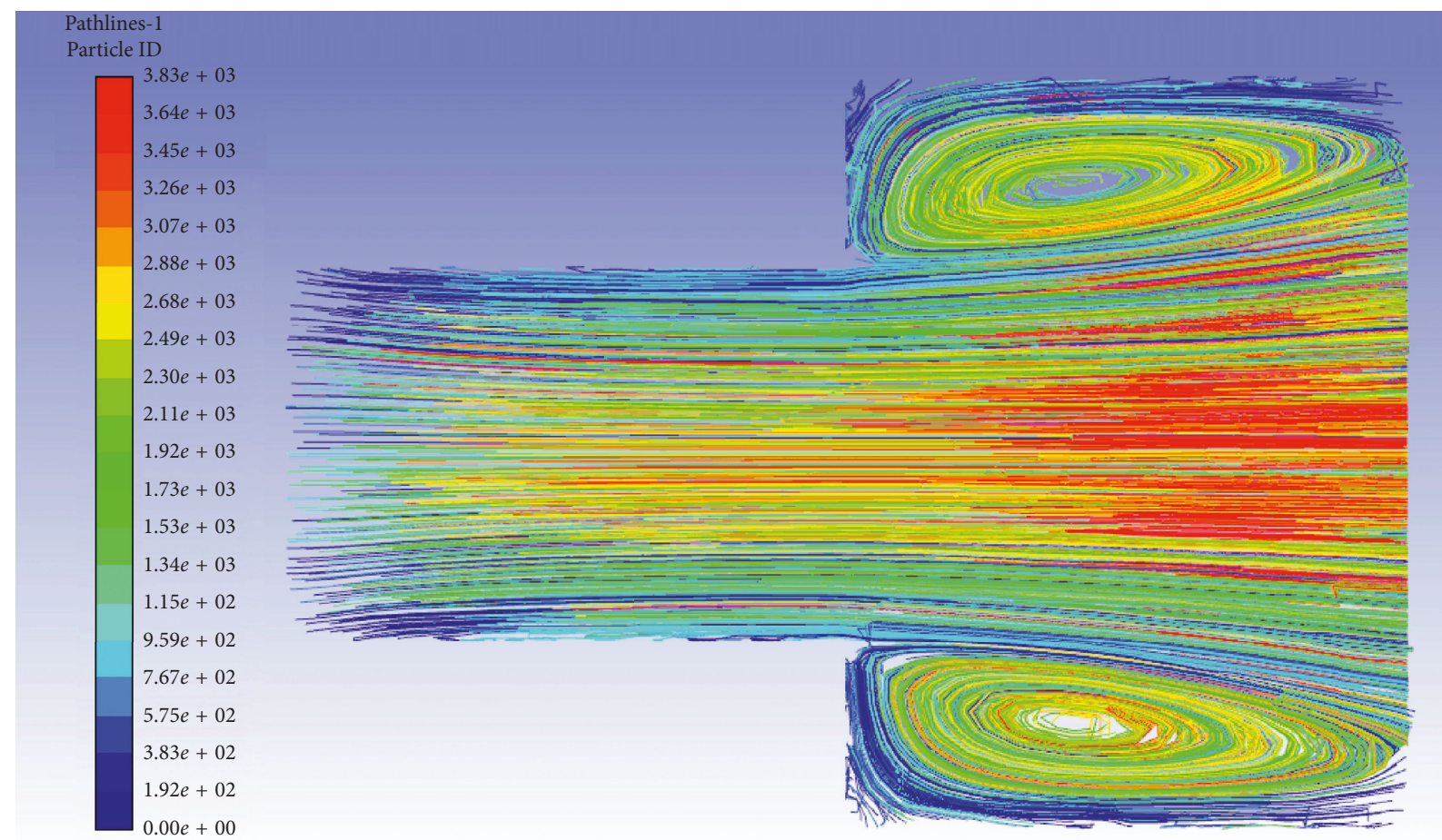

Figure 11: Sudden extension ratio is 2.

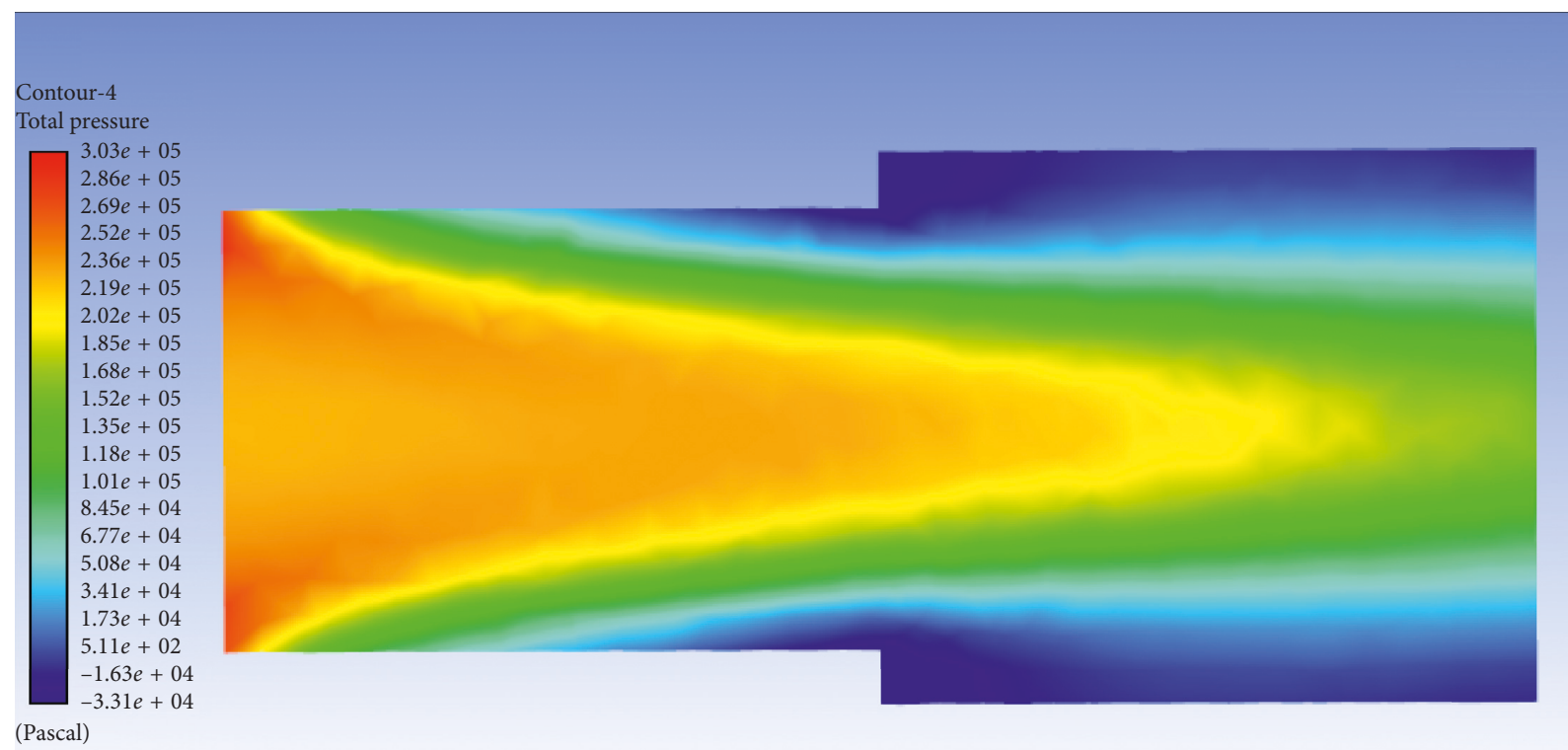

Figure 12: Pressure nephogram (sudden expansion ratio is 1.25).

cannot be bent, which will cause the vortex at the sudden extension of cross section and then cause pressure losses and energy losses. The flow coefficient of the sudden extension of cross section with chamfer is 0.47 , and that of the sudden extension of cross section without chamfer is 0.49 , which increases the flow capacity by $4.25 \%$.
Sudden spread with chamfer will make the streamline at the sudden extension of cross section more smoothly, which is conducive to reducing the size of the vortex, reducing the velocity and turbulent kinetic energy in the wall reflux zone, and reducing pressure losses and energy losses. 


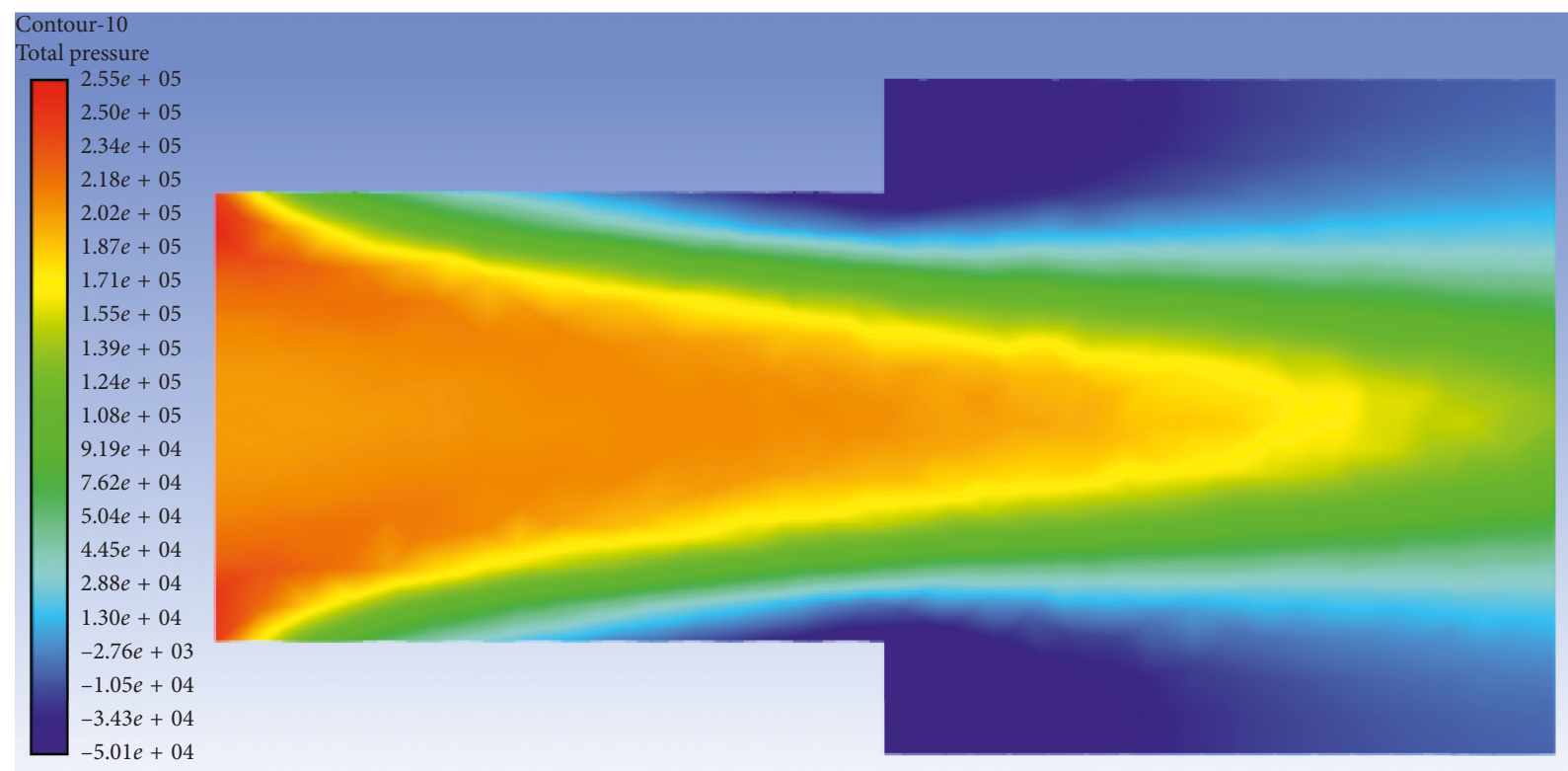

(Pascal)

Figure 13: Pressure nephogram (sudden expansion ratio is 1.5).

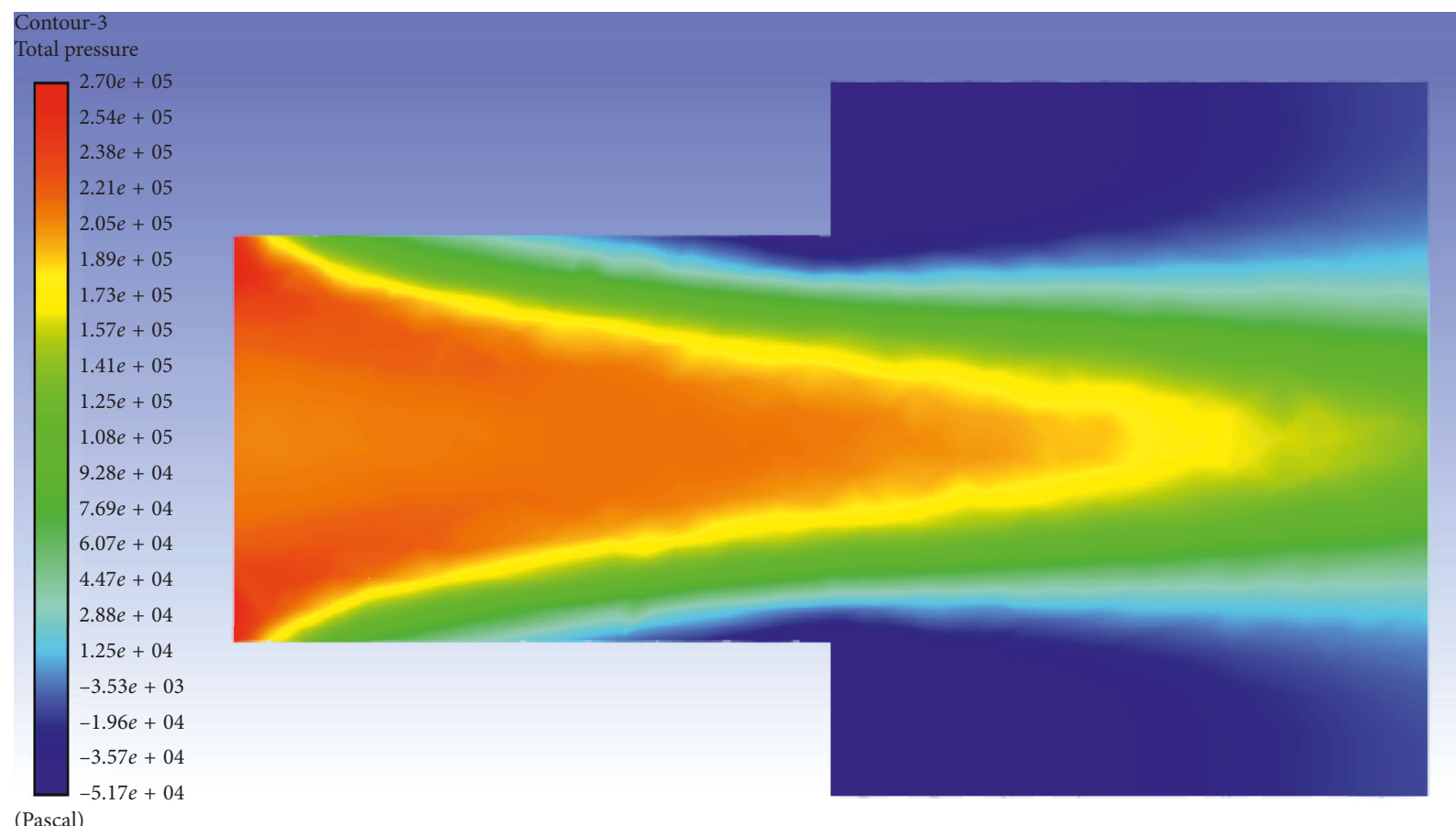

Figure 14: Pressure nephogram (sudden expansion ratio is 1.75).

2.5. Effect of Core Angle on Energy Losses of Poppet Valve. From Figures 25-27, it can be seen that the angle of the poppet valve core is inversely proportional to the size of the vortex at valve port and the edge of valve port at the same opening height. From Figures 28-30, it can be seen that there is a fluid separation phenomenon at the tip of the poppet valve core. The larger the angle of the poppet valve core is, the weaker the fluid separation phenomenon is. The larger the angle of the core, the larger the high pressure zone at the core outlet. From Figures 31-35, it can also be seen that with the increase of the core angle, the turbulent kinetic energy region decreases, the turbulent intensity decreases, and the 


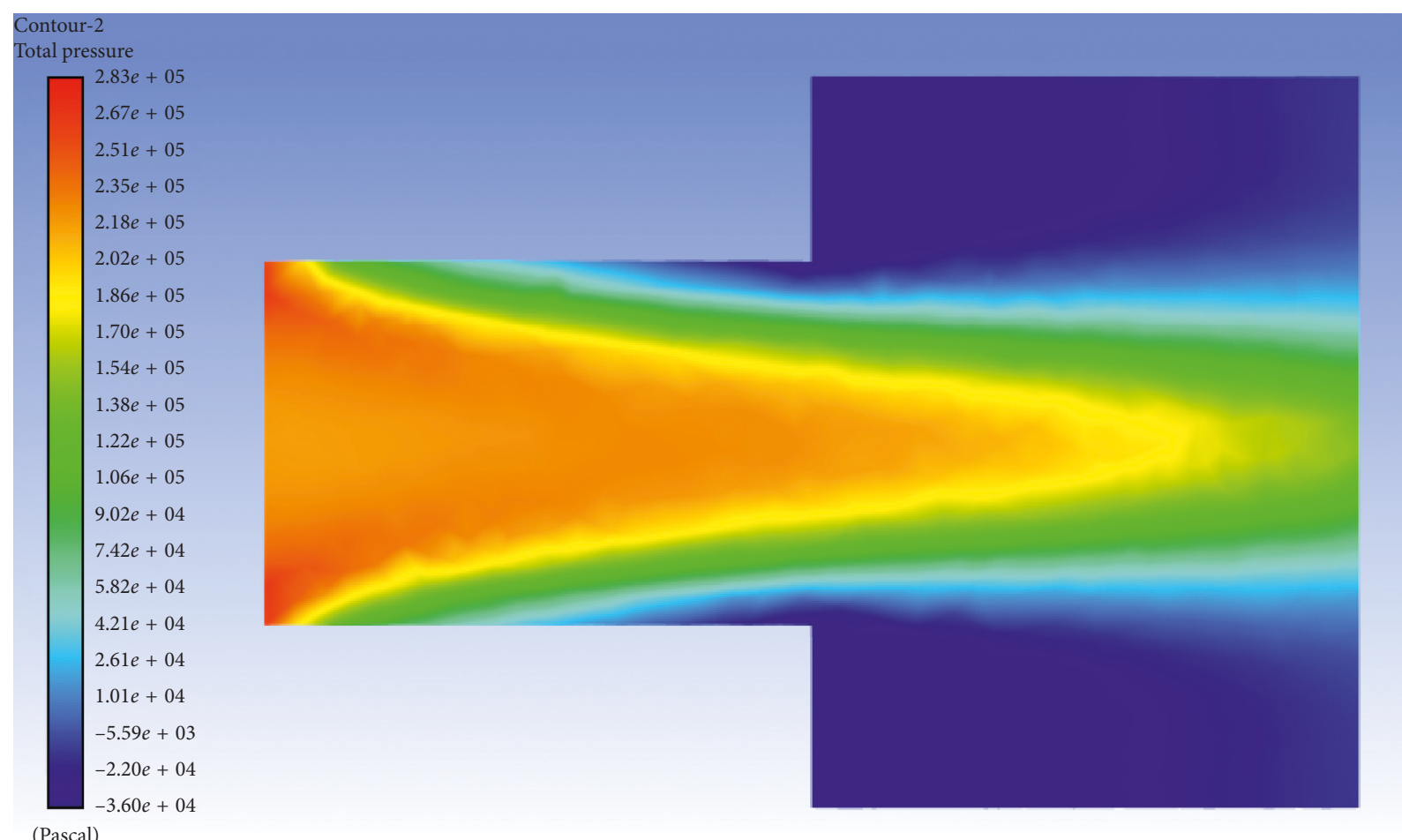

Figure 15: Pressure nephogram (sudden expansion ratio is 2).

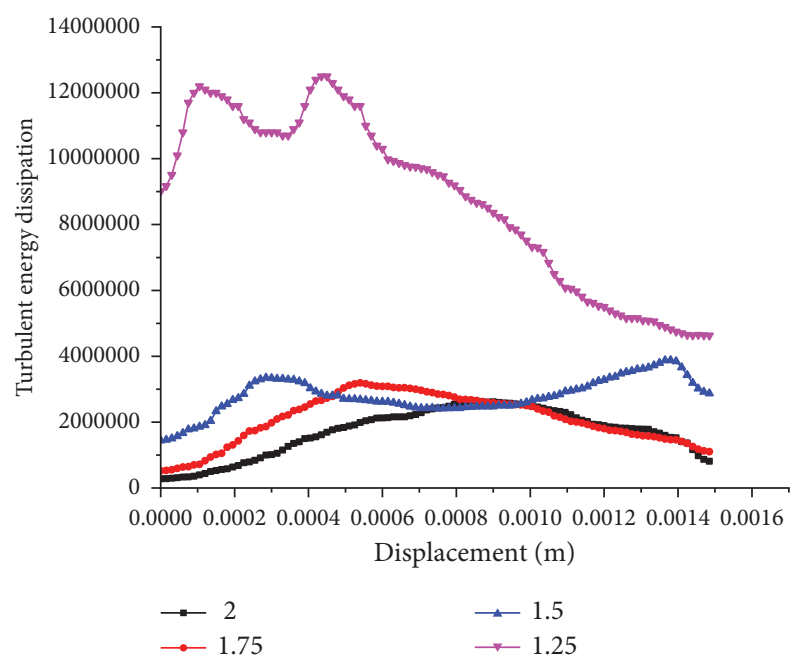

FIGURE 16: Turbulent energy distribution at the sudden extension of cross section.

energy losses in the region decrease. With the increase of the angle of the core, the negative pressure on the wall of poppet valve becomes smaller and the flow velocity decreases, and the pressure change rate and the flow velocity change rate become smaller. Large negative pressure will accelerate the vortex velocity in the wall reflux zone, and the flow field changes more complex, resulting in greater pressure losses and energy losses.

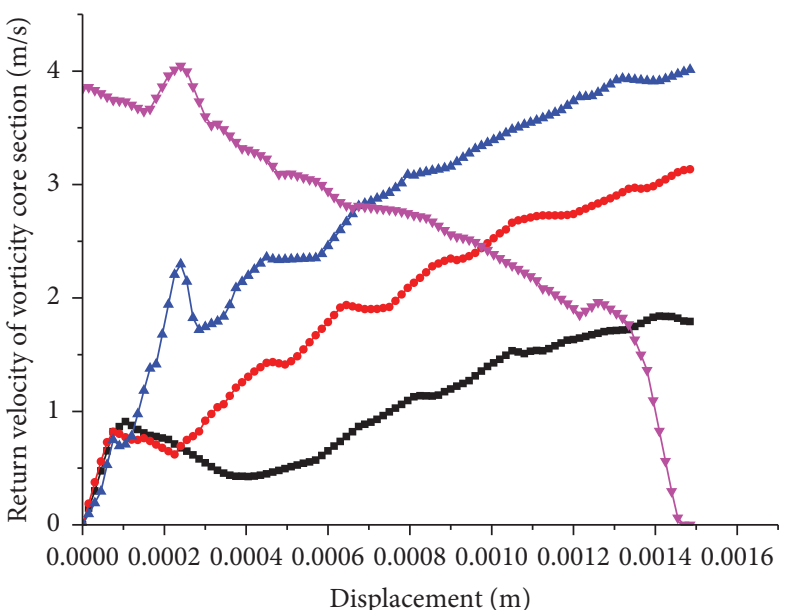

$$
\begin{array}{ll}
-2 & \longrightarrow 1.5 \\
-1.75 & \rightarrow 1.25
\end{array}
$$

FIGURE 17: Velocity distribution in the recirculation zone of vortex core section.

In summary, energy losses at the poppet valve port include the energy losses at the edge of the valve port and energy losses at the tip of the valve core. When the poppet valve core is opened at the same height, the smaller the angle of the poppet valve core is, the faster the fluid flows through the poppet valve core, which accelerates the vortex flow in the edge area of the valve port, enhances the turbulence 


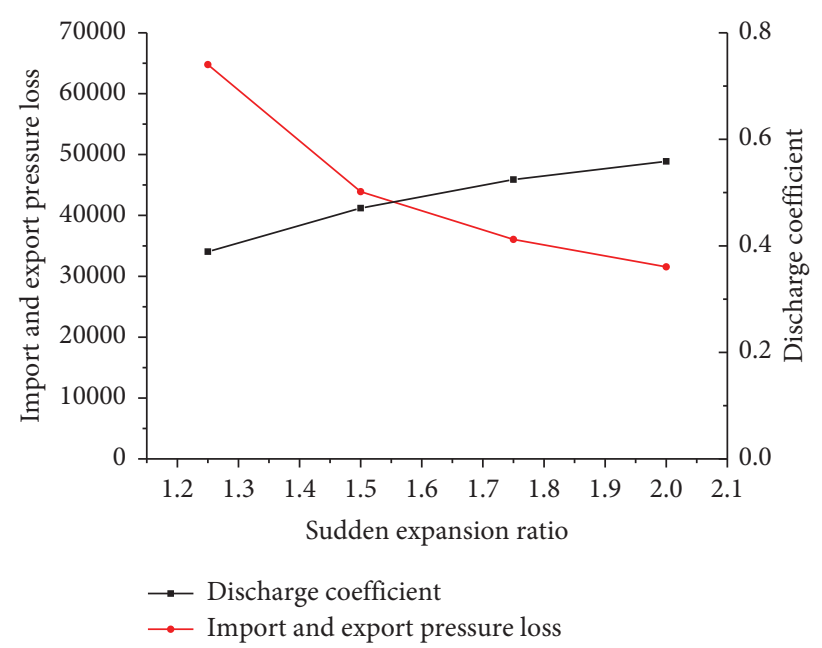

FIGURE 18: Flow coefficient of different sudden extension ratios and the pressure difference between import and export.

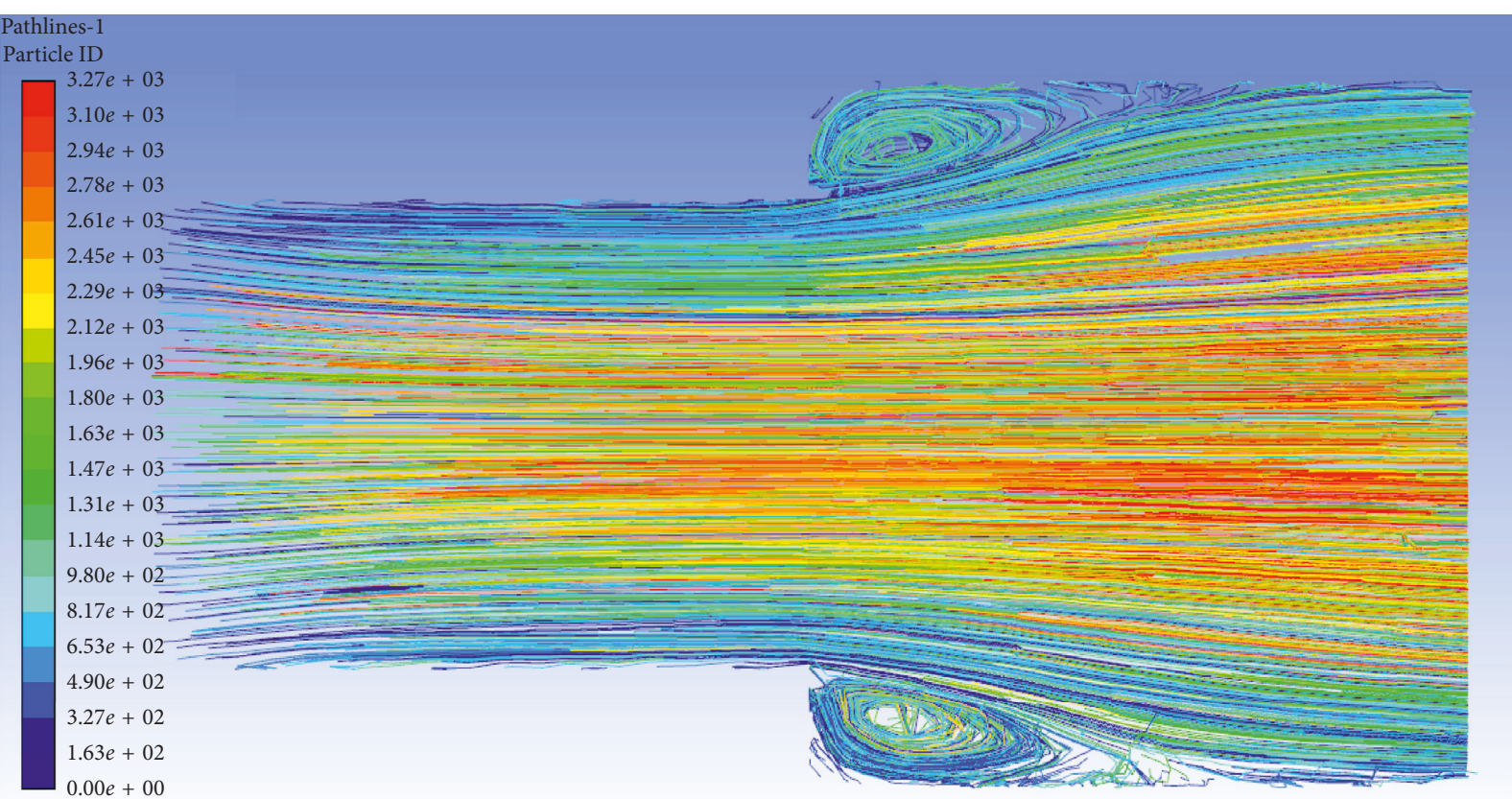

FIGURE 19: Without chamfer (sudden spread ratio is 1.5 ).

intensity, and increases the energy losses. The flow separation phenomena will occur in the tip area of the poppet valve core with circular cross section, which makes the separation vortex occur in the tip area of the valve core and increases its energy losses. With the increase of the angle, the energy losses decrease.

\section{Control Model of Energy Dissipation Process}

According to the energy dissipation model of the whole system proposed in Section 2, it can be seen that the key factors are as follows: pulse width modulation (PWM) of the digital damper unit, number of digital damper units, chamfer on the sudden extension of cross section of the digital damper unit, sudden extension of cross section with different ratios of the digital damper unit, and cone spool with different angles of the digital damper unit. All of them affect the energy dissipation process.

Theoretically, the above five parameters should be controlled during the process of the digital damper. Theoretically, the above five parameters should be controlled during the damping process. In this paper, PWM and number of digital damper units are the present adjustable parameters. Only the PWM of digital damping elements and the number of digital damping elements are designed as the adjustable parameters at present.

The work equation is as follows: $W=F S$. Under the same energy absorption situation, the force on the piston 


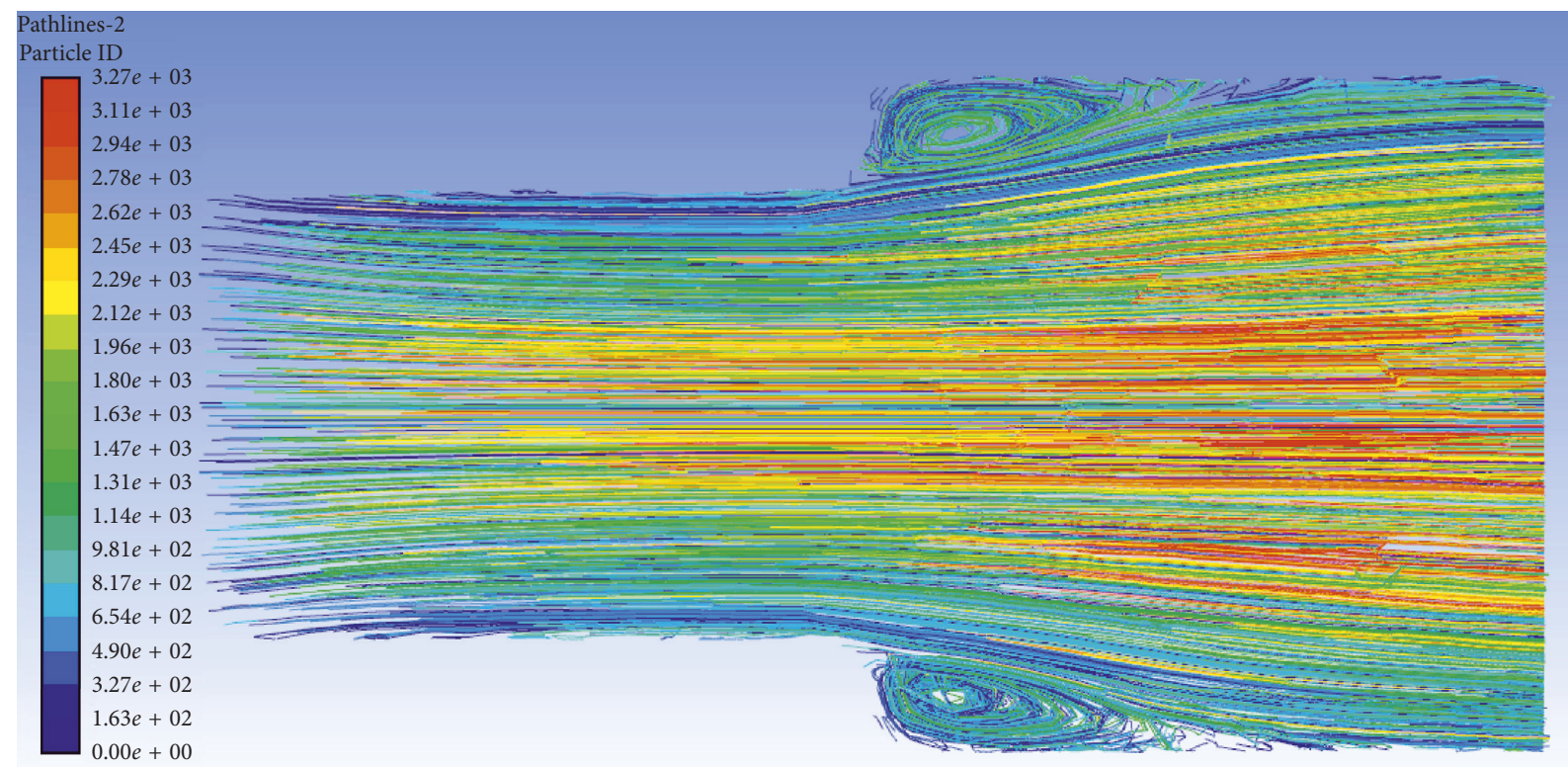

Figure 20: With chamfer (sudden spread ratio is 1.5 ).

TABle 5: Sudden spread ratio of 1.5 with or without chamfer reflux zone.

\begin{tabular}{lcccc}
\hline Entry condition & Type & Center location of vortex & Vortex structure & Length of reflux zone \\
\hline \multirow{2}{*}{$15 \mathrm{~m} / \mathrm{s}$} & Without chamfer & $\mathrm{O}_{1}(0.18 D, 0.16 D)$ & Stable reattachment vortex & $0.94 D$ \\
& With chamfer & $\mathrm{O}_{1}(0.2 D, 0.13 D)$ & Stable reattachment vortex & $0.8 D$ \\
\hline
\end{tabular}

$D$ is the diameter of the damping hole.

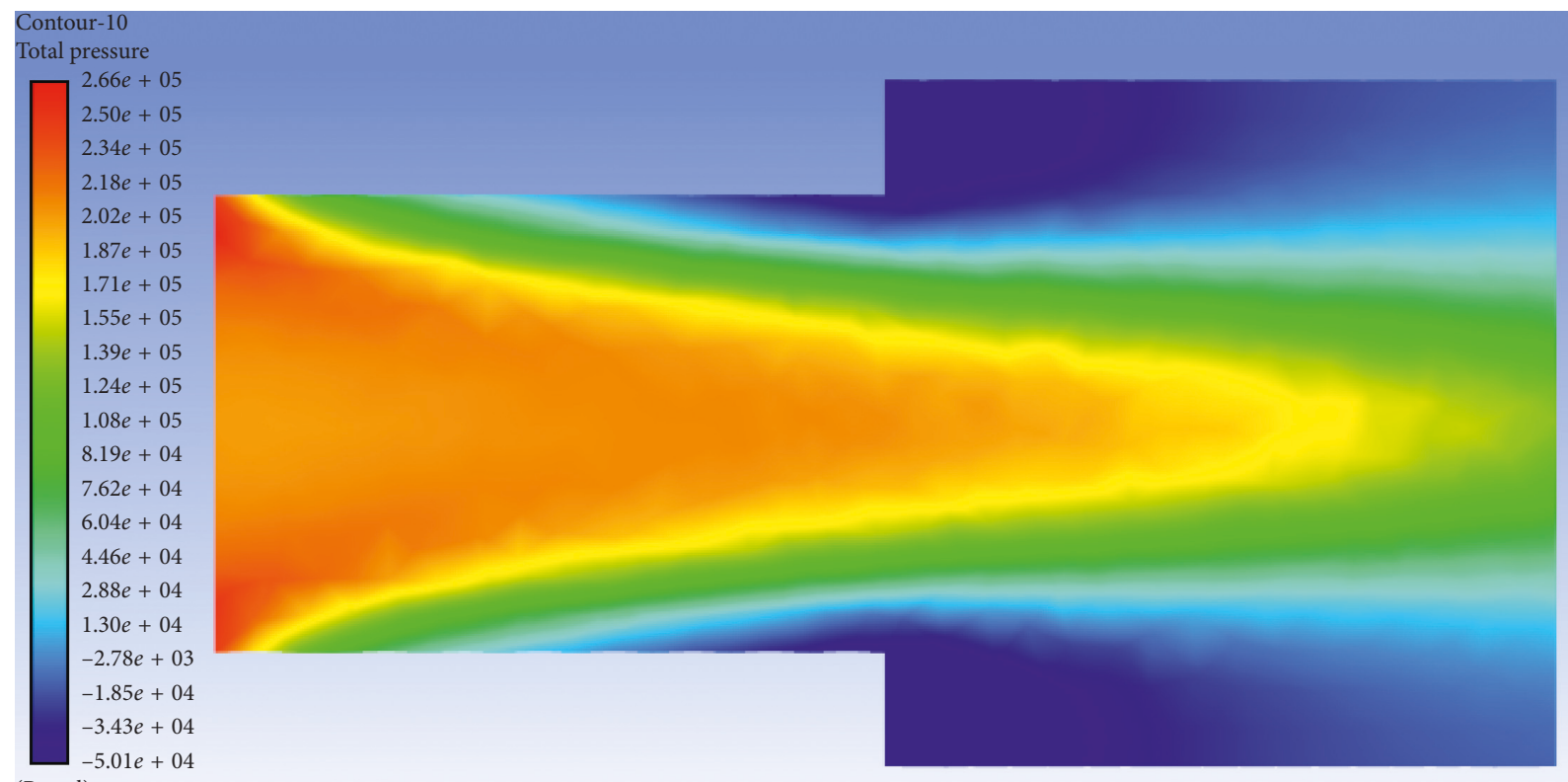

Figure 21: Pressure nephogram without chamfer (sudden spread ratio is 1.5).

rod of the damper is inversely proportional to the displacement of the piston in the damper cylinder. In the formula, $W$ is the total energy absorbed by the hydraulic damper, $F$ is the force on the piston rod in the damper cylinder during the buffering process, and $S$ is the displacement of the piston in the damper cylinder during the buffering process.

Two control modes are designed for the digital hydraulic damper, which are the minimum pressure mode and the minimum displacement mode. For the minimum pressure 


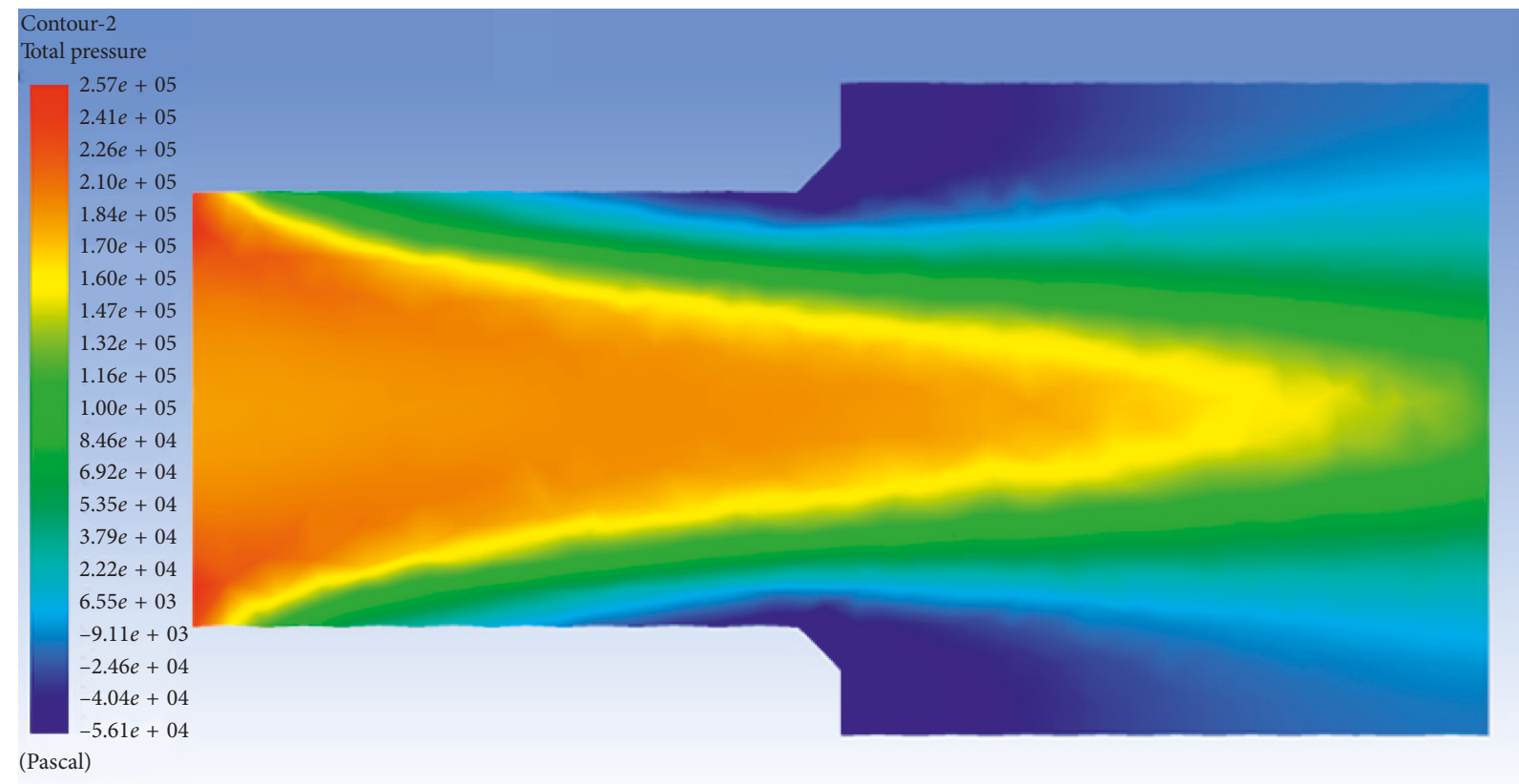

Figure 22: Pressure nephogram with chamfer (sudden spread ratio is 1.5).

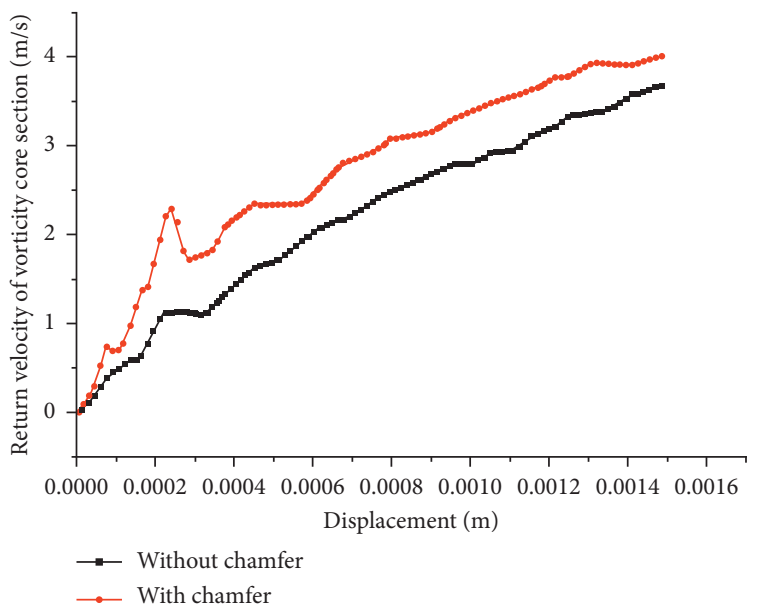

Figure 23: Velocity distribution in the reflux zone of vortex center.

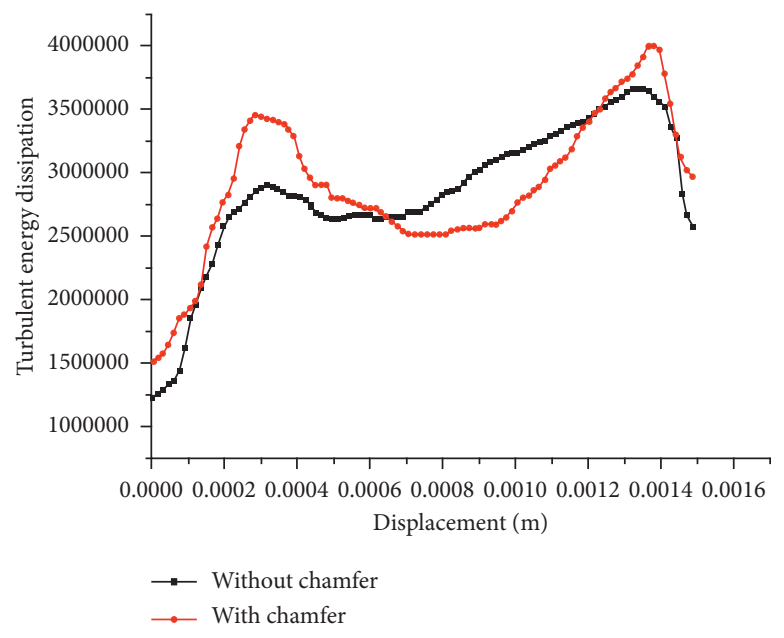

Figure 24: Distribution of turbulent kinetic energy at extension of cross section. 


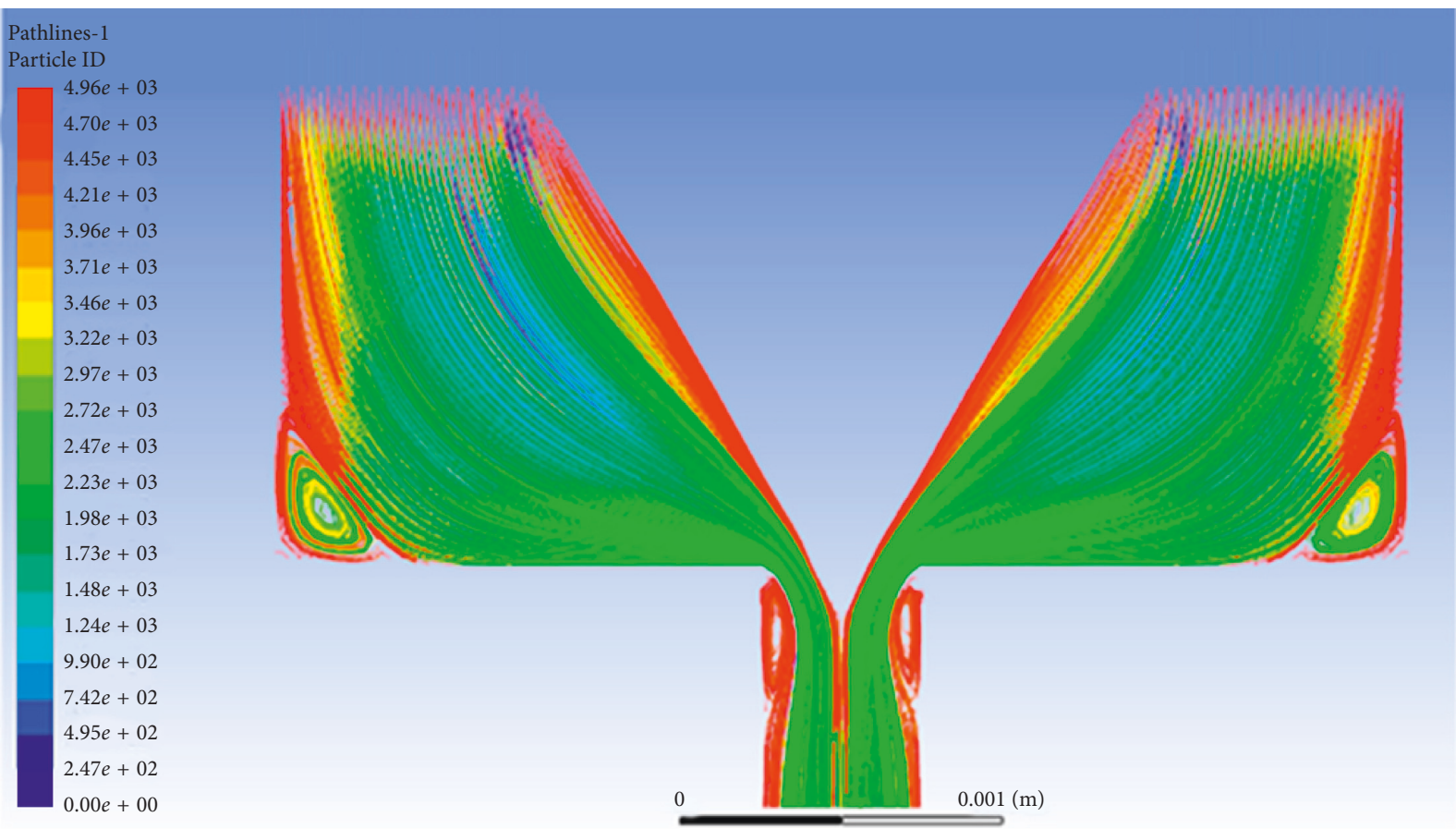

Figure 25: Flow streamline when the poppet valve core angle is $60^{\circ}$.

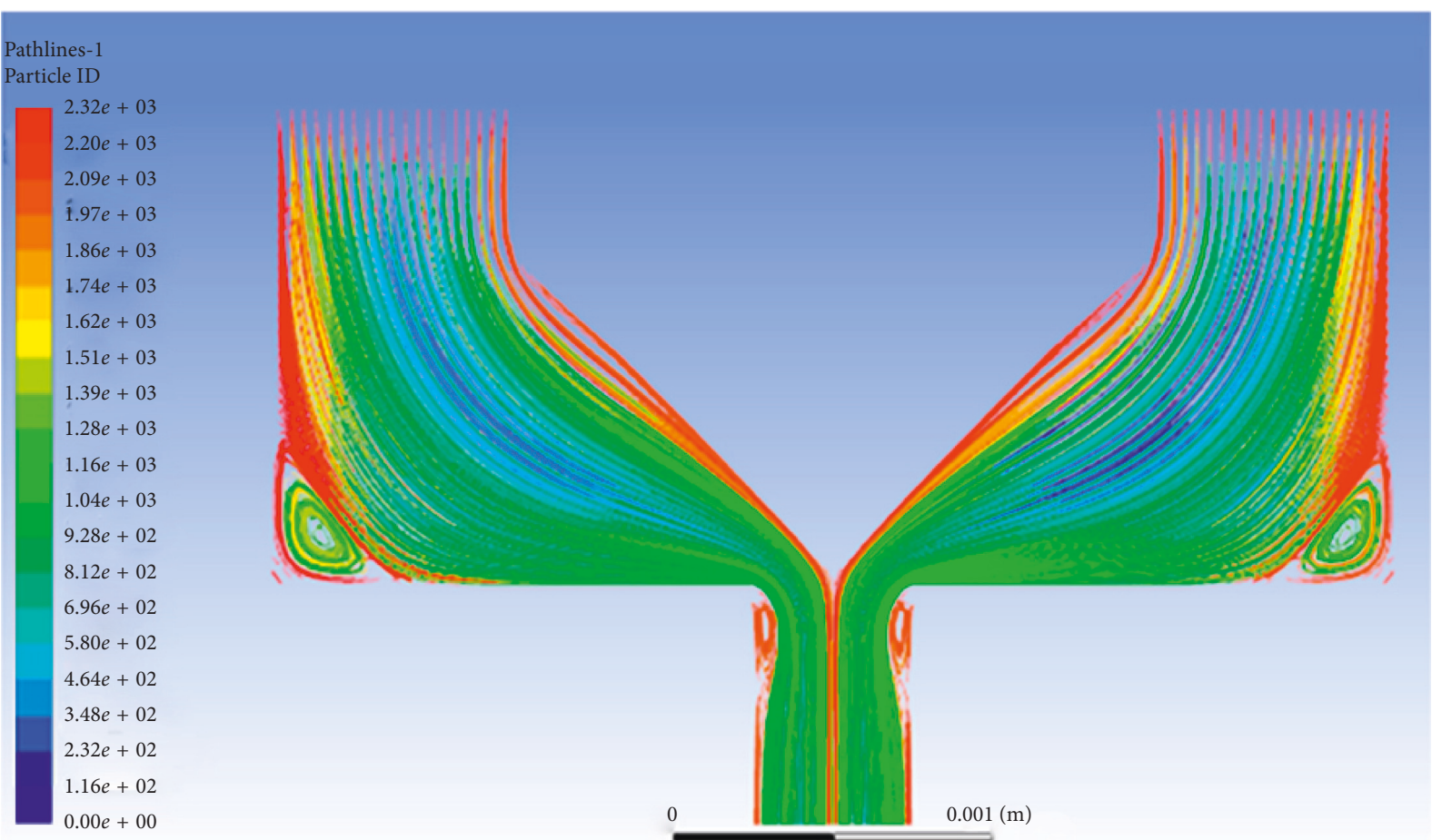

Figure 26: Flow streamline when the poppet valve core angle is $90^{\circ}$.

mode, the force on the piston rod in the damper cylinder should be minimal under the situation of maximum allowable displacement. For the minimum displacement mode, the displacement of the piston in the damper cylinder should be minimal under the situation of maximum allowable stress.
The energy dissipation process of the whole system is based on different working modes so as to determine the appropriate buffering pressure value. Here, the buffering pressure value is described with $F$ in Figure 36. The digital hydraulic damper is equipped with speed sensor and 


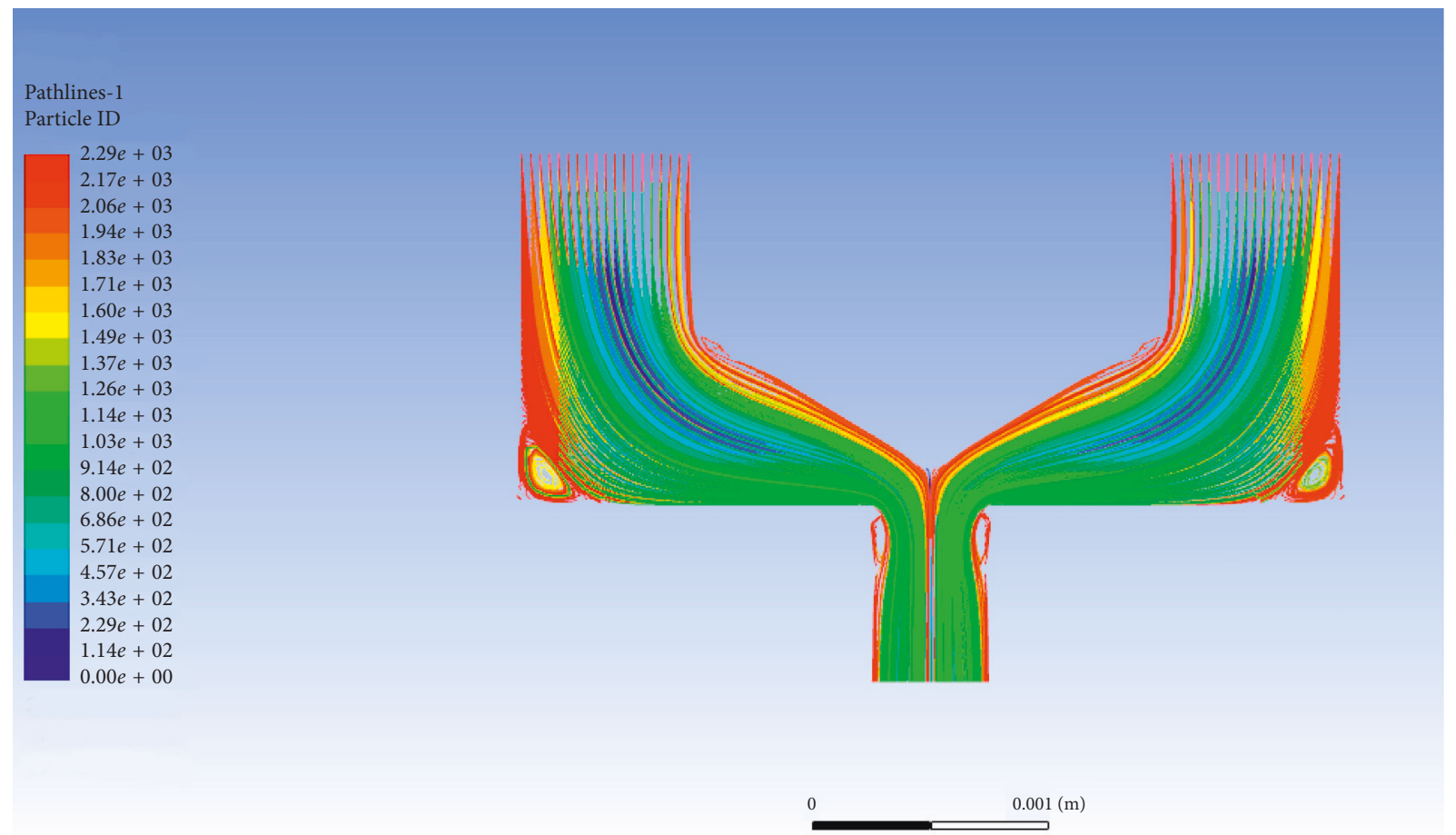

Figure 27: Flow streamline when the poppet valve core angle is $120^{\circ}$.

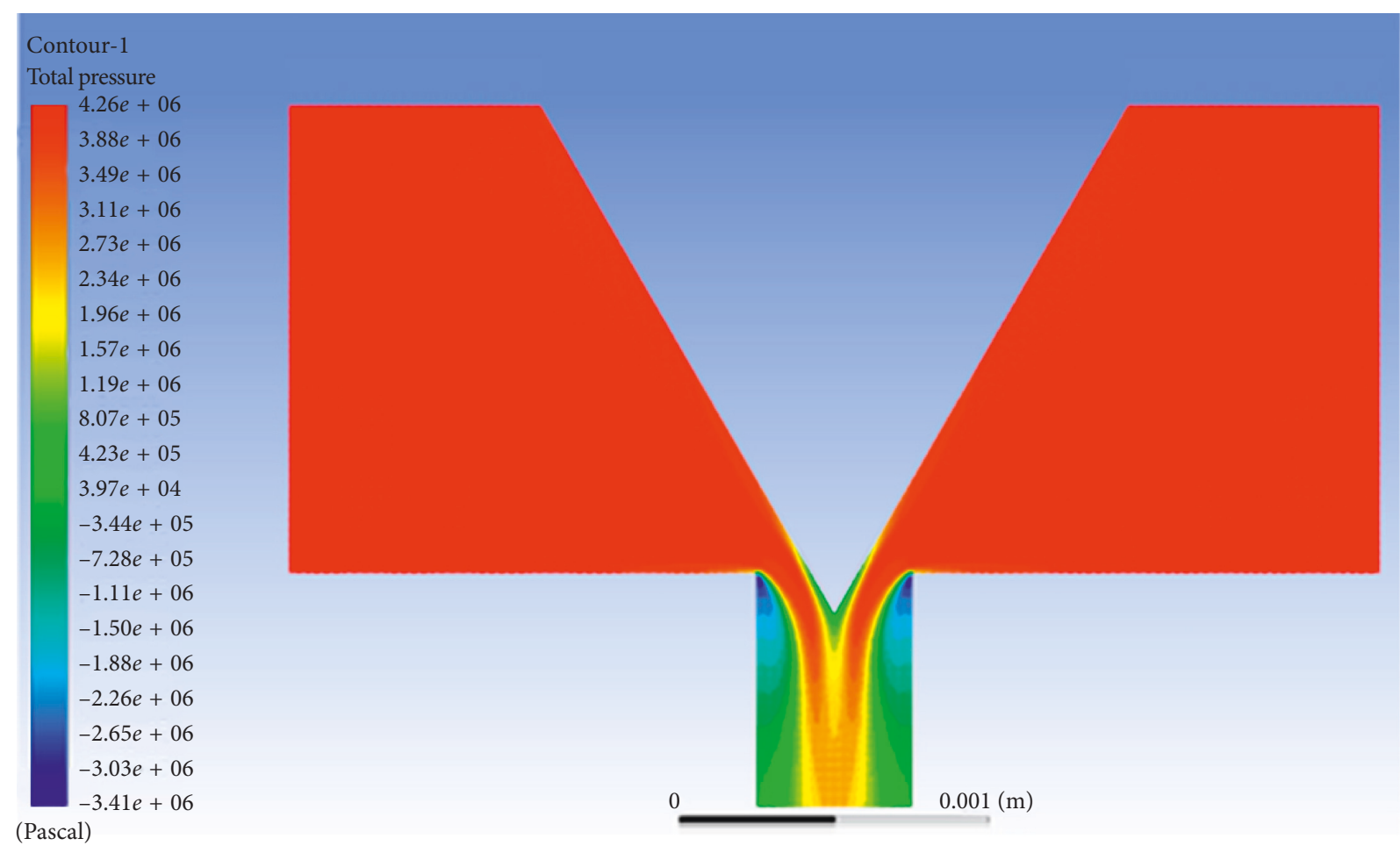

Figure 28: Pressure nephogram of a poppet valve core angle of $60^{\circ}$.

pressure sensor that are used to detect the impact velocity and the pressure in the rodless chamber of the damper cylinder, respectively. The speed of the whole moving parts is described with $V$ in Figure 36.
The control process is shown in Figure 36. When the impacting body contacts the piston rod of the digital hydraulic damper, the velocity of the piston, piston rod, and impacting body will be the same. The velocity of the impacting body is 


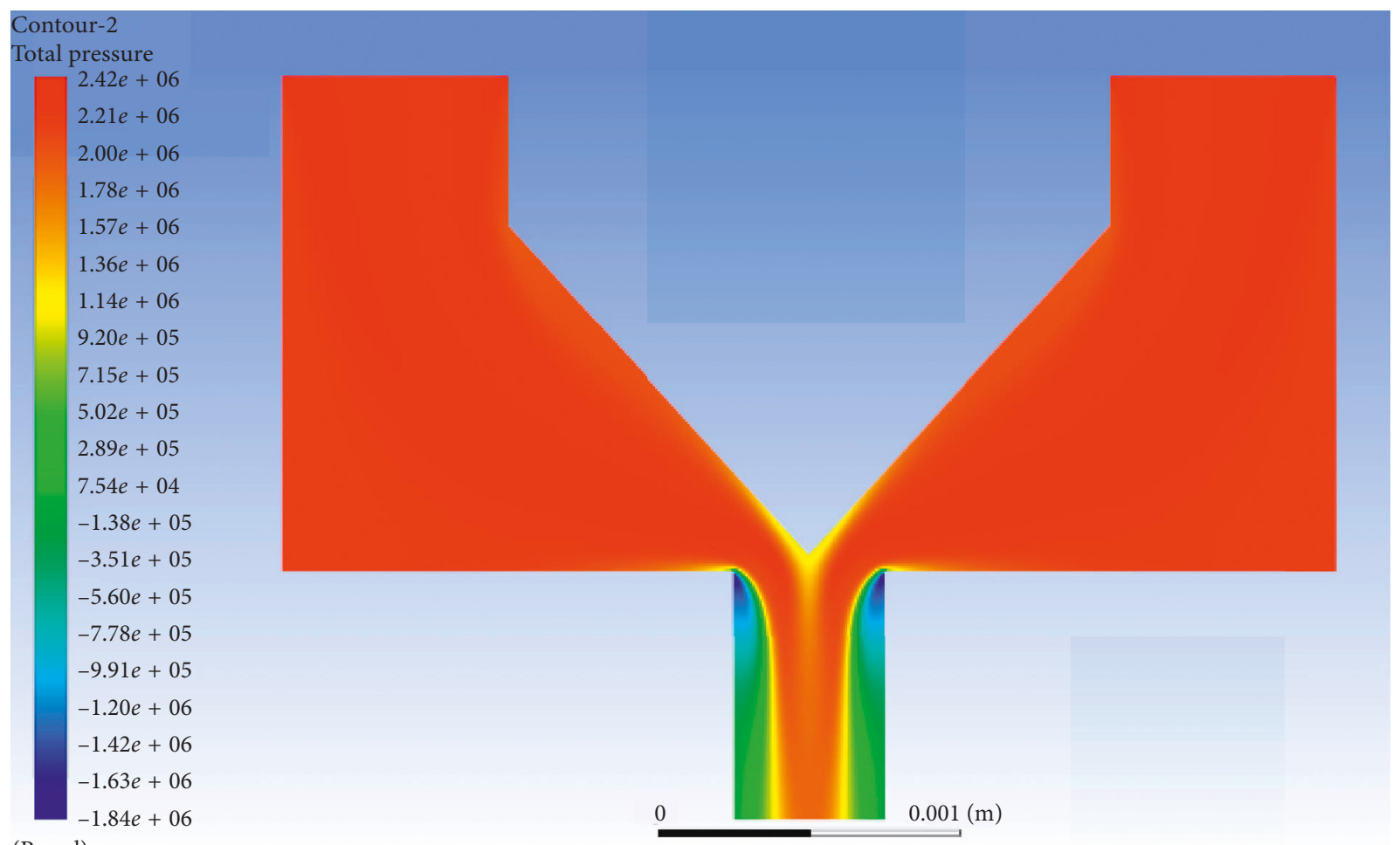

(Pascal)

Figure 29: Pressure nephogram of a poppet valve core angle of $90^{\circ}$.

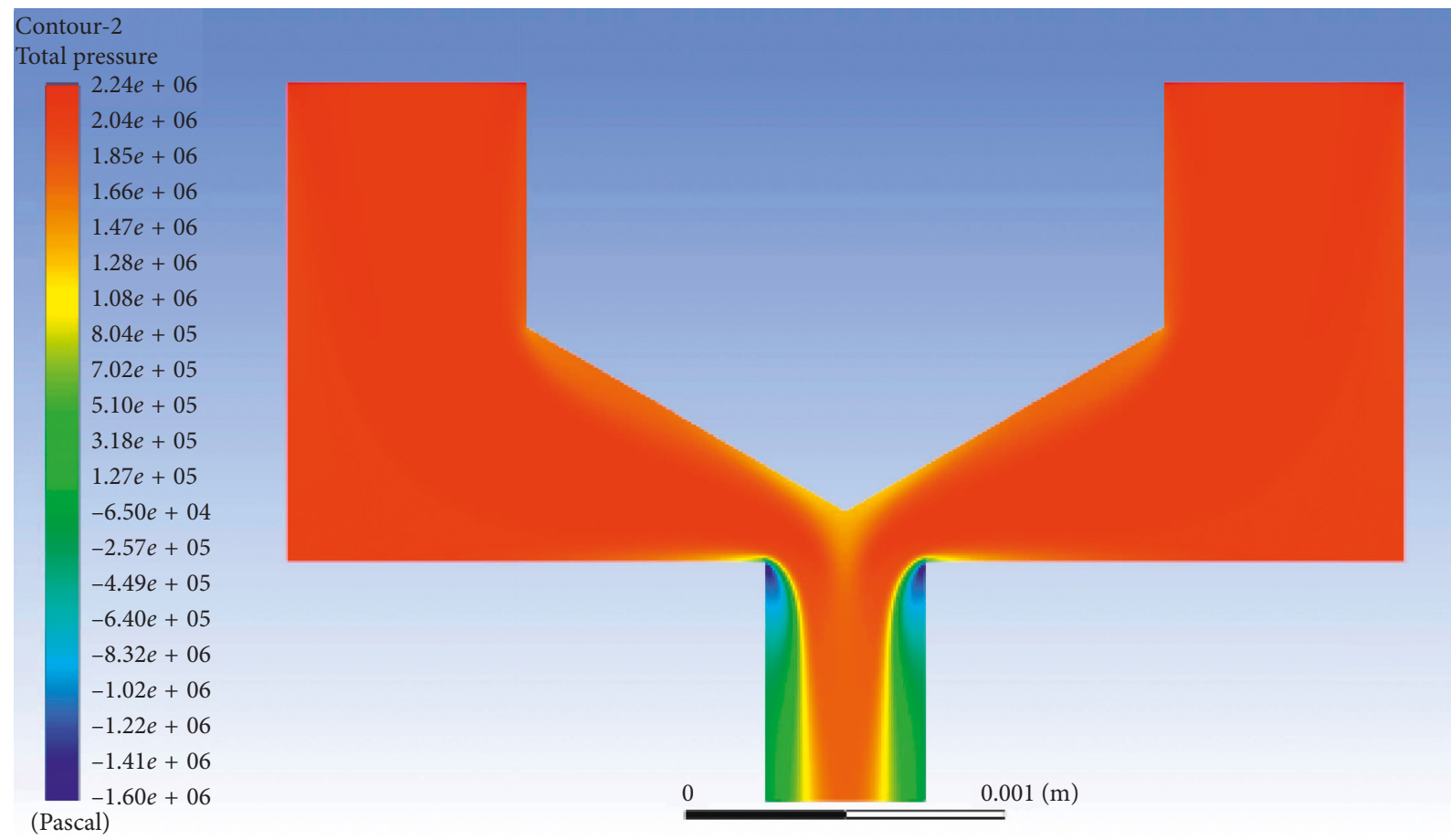

Figure 30: Pressure nephogram of $120^{\circ}$ flow field.

detected by the velocity sensor in real time. The pressure of the rodless chamber of the damper cylinder is detected by the pressure sensor in real time. After the averaging value processing, PWM of the digital damping unit and numbers of the digital damping units are controlled by the digital throttling control algorithm. According to the digital 


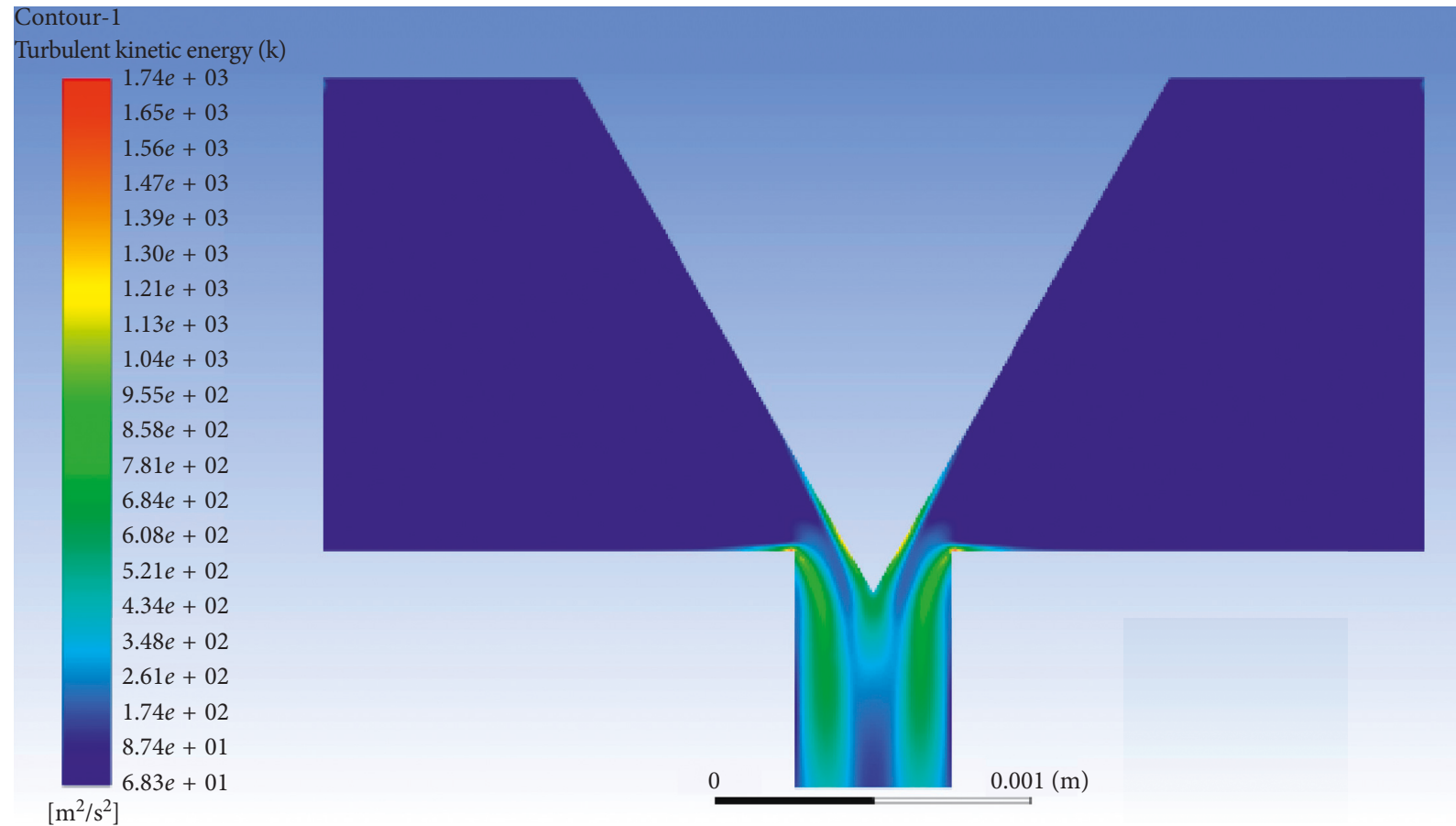

FIgURE 31: Turbulent kinetic energy distribution nephogram of a poppet valve core angle of $60^{\circ}$.

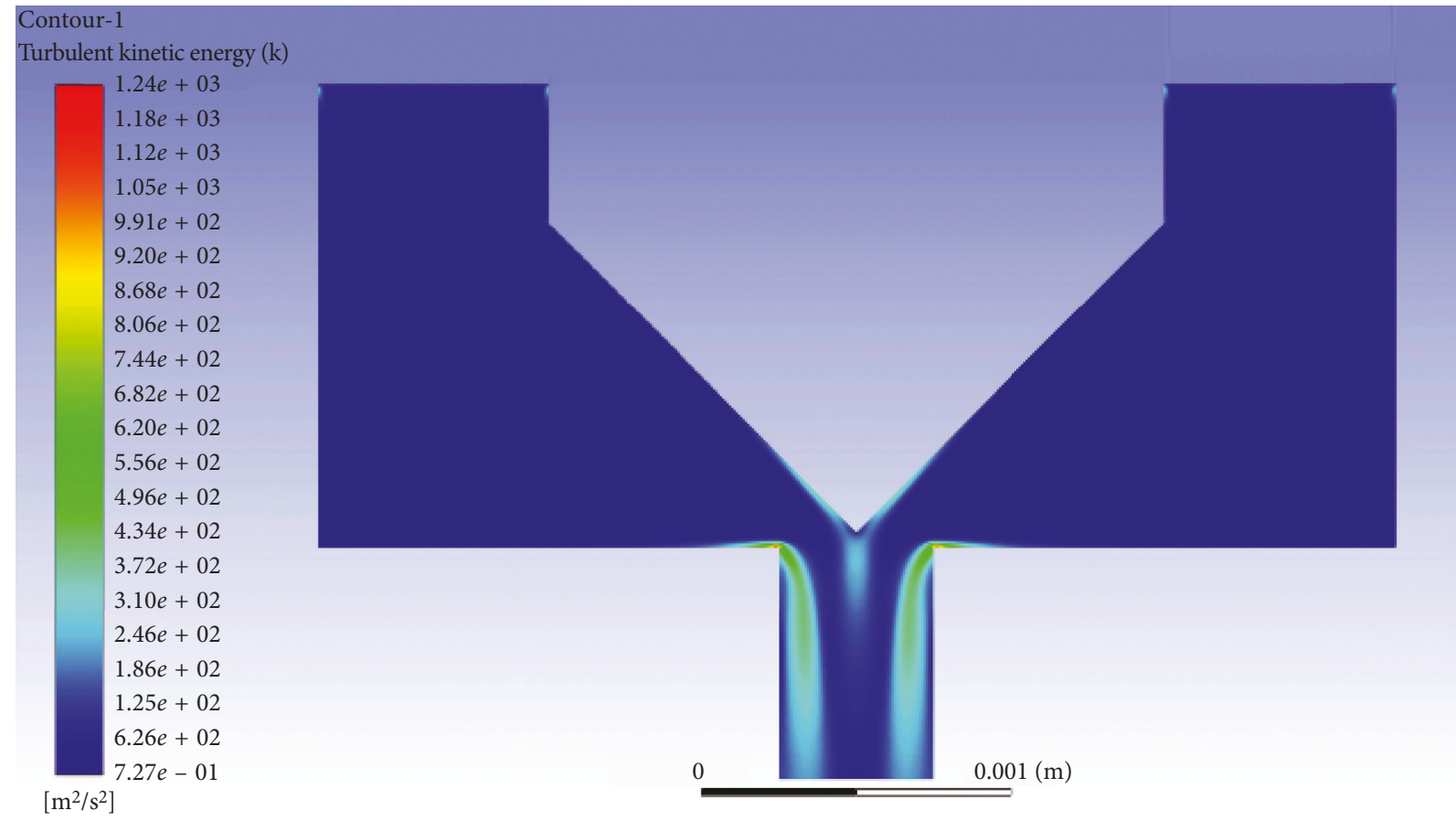

FIGURE 32: Turbulent kinetic energy distribution nephogram $\left(90^{\circ}\right)$.

throttling control algorithm, the throttle area of the device can be adjusted in real time according to the moving situation of the impacting body so as to obtain the best total throttle area. Then, the value of the pressure and the buffering displacement of the digital hydraulic damper can be adjusted so as to achieve the purpose of smooth control and timely buffering.

\section{Conclusion}

In this paper, the mathematical model of energy losses of digital damper unit and the energy dissipation model of the whole digital damper are established. Based on the energy dissipation model, the energy dissipation mechanism of a 


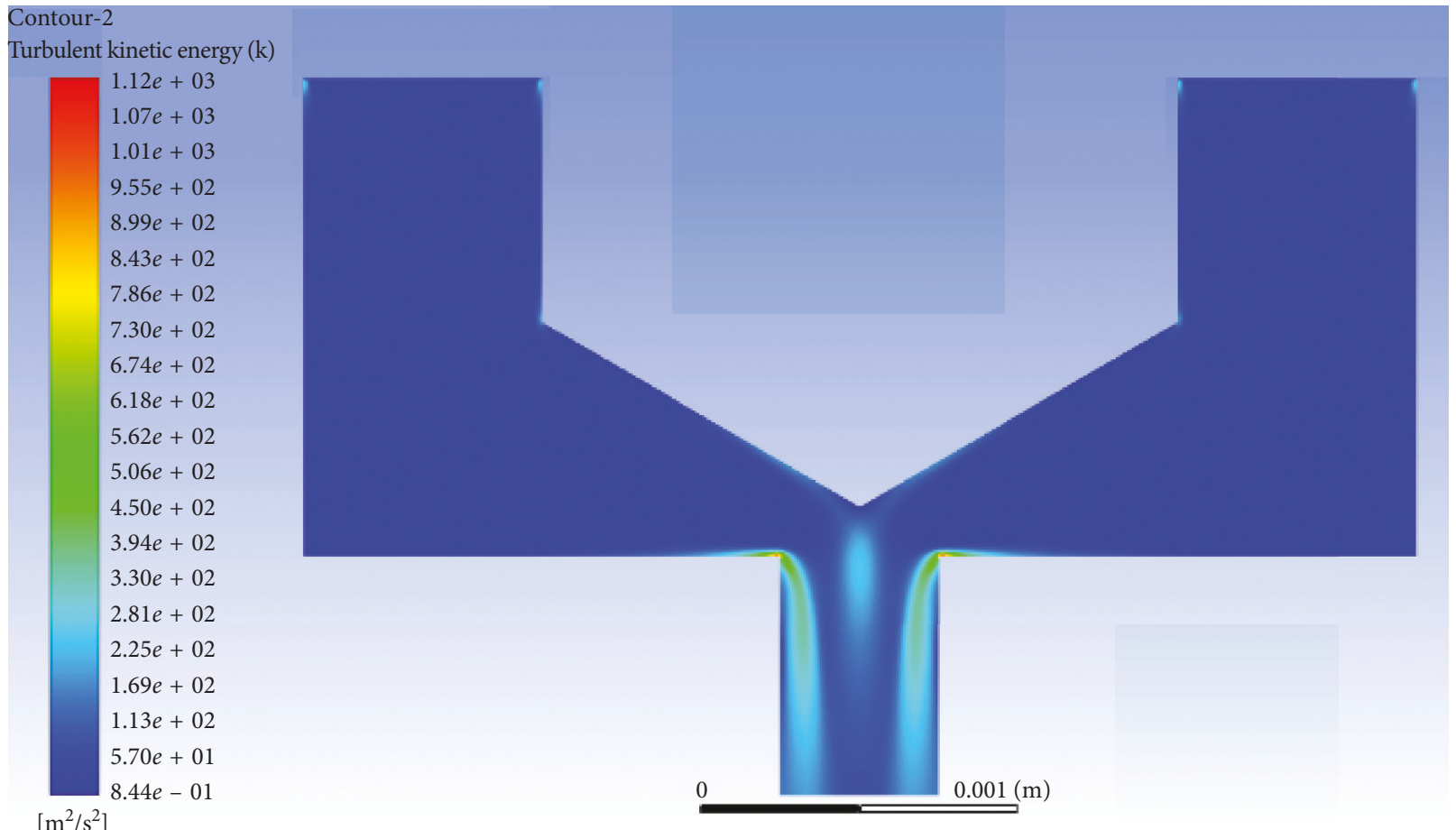

Figure 33: Turbulent kinetic energy distribution nephogram $\left(120^{\circ}\right)$.

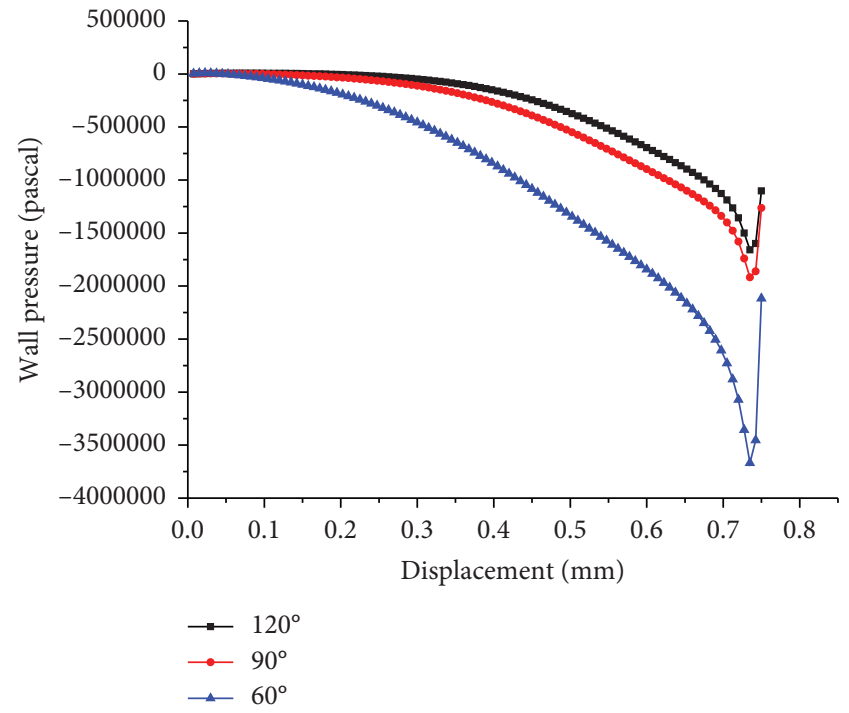

Figure 34: Pressure distribution on the wall of poppet valve port.

single digital damper unit is studied. The effect on energy losses caused by the three elements of the digital damper, which are local pressure losses at sudden change cross section, frictional pressure losses through the orifice, and local pressure losses through the cone valve port, is analyzed. On the basis of the study of energy dissipation mechanism, the control model of the energy dissipation process is given, which lays a theoretical foundation for the realization of better effect during the buffering process.

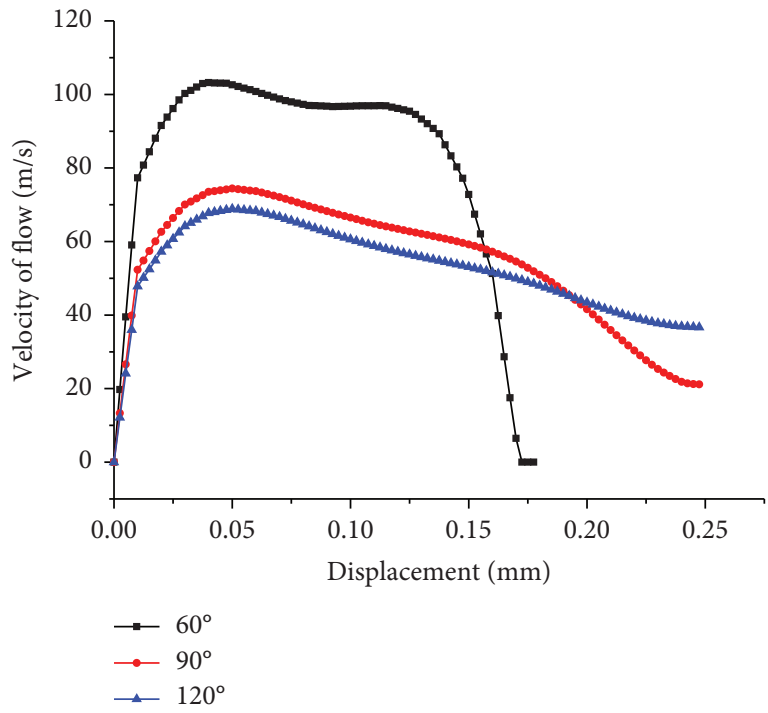

FIgURE 35: Velocity distribution at poppet valve port.

In the future works, the effect on energy losses, which is caused by local pressure losses at sudden change cross section, frictional pressure losses through the orifice, and local pressure losses through the cone valve port, will be tested physically by means of a test rig so as to verify the simulation models. An optimized structure of the digital damper unit will be given according to the testing and simulation data, and an optimized energy dissipation control process model will be given. 


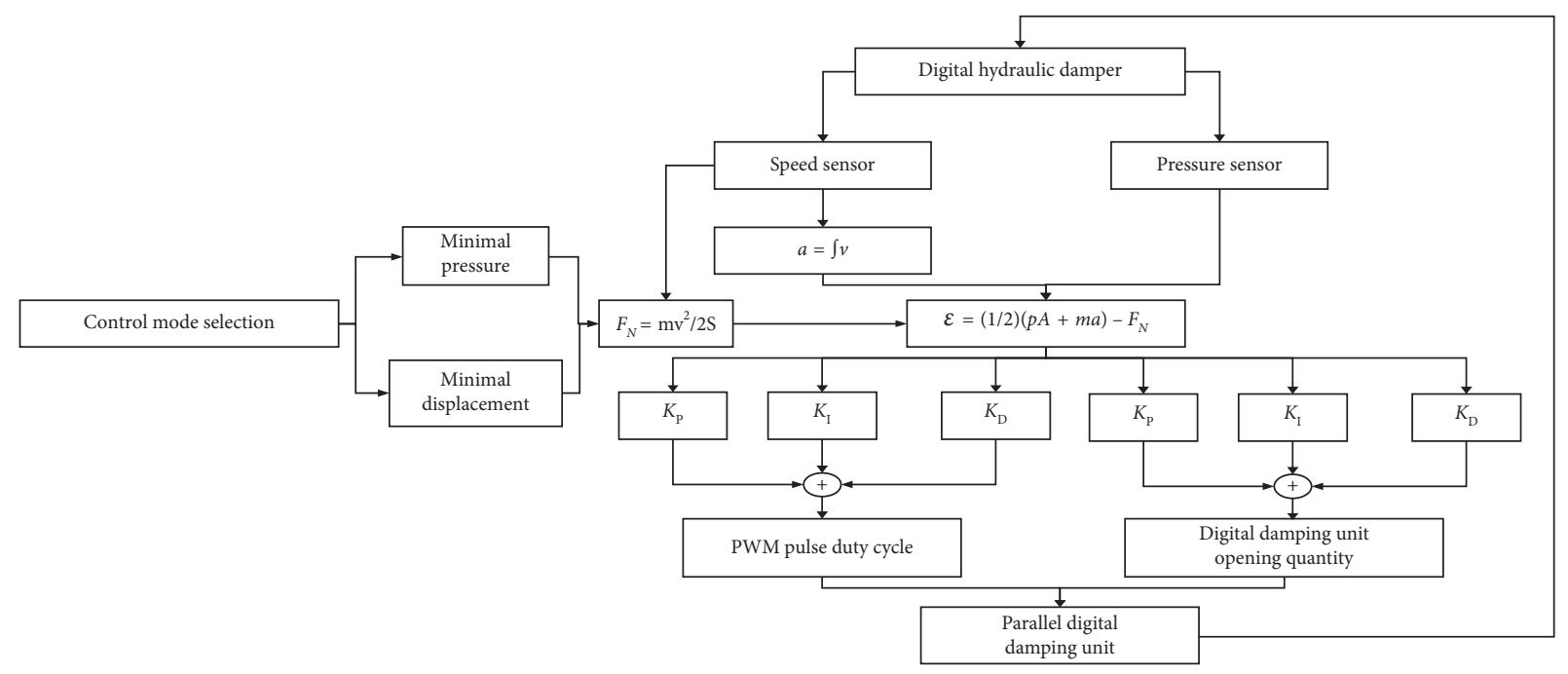

FIGURE 36: Control model of the energy dissipation process.

\section{Data Availability}

The data used to support the findings of this study are included within the article.

\section{Conflicts of Interest}

The authors declare that they have no conflicts of interest.

\section{Acknowledgments}

This work was supported by the Natural Science Foundation of Shandong Province (ZR2016EEM02), the National Natural Science Foundation of China (51375282), and the State Key Laboratory of Mining Disaster Prevention and Control cofounded by Shandong Province and the Ministry of Science and Technology (51010305029).

\section{References}

[1] L. Jiang, Gradual Throttling Hydraulic Absorber Structural Design Optimization and Researchon Dynamic Characteristics, Shenyang Ligong University, Shenyang, China, 2016.

[2] C. Wang, Z. Qiu, Q. Zeng, and T. Wang, "Design and buffering characteristics study of a porous hydraulic buffer," Machine Tool and Hydraulics, vol. 46, no. 9, pp. 93-96, 2018.

[3] P. Wang and G. Shen, "Energy absorption performance of the multi-unit VehicleImpact on clay buffer," Urban Mass Transit, vol. 19, no. 6, pp. 80-85, 2016.

[4] M. Qi, Study on Dynamic Characteristics of Viscoelastic Buffer, Nanjing University of Science and Technology, Nanjing, China, 2014.

[5] D. Wang, T. Su, H. Wang et al., "The influence of the viscoelatic elastomer damper to the firing accuracy of the large caliber machine gun," Journal of Ordnance Equipment Engineering, vol. 38, no. 12, pp. 13-16, 2017.

[6] H. A. O. Baochen, W. Sun, and X. Pan, "Study on the buffer characteristics of artillery hydraulic buffer," Journal of Ordnance Equipment Engineering, vol. 39, no. 9, pp. 54-58, 2018.

[7] C.-L. Wang, Z.-W. Qiu, Q.-L. Zeng, Y.-X. Liu, and G.-Y. Miao, "An active-control digital hydraulic damper: design, modeling and simulation," in Proceedings of the 11th International Fluid Power Conference, Aachen, Germany, March 2018.

[8] J. I. Hong, X. fu, and H. Yang, "Analysis and calculation on typical shape orifice areas in hydraulic valves," Machine Tool \& Hydraulics, no. 5, p. 1416, 2003.

[9] J. I. Hong, D.-S. Wang, D. Ding, Z. Tan, and X. Liu, "Calculation method of orifice area of spool valve with notches," Journal of Lanzhou University of Technology, vol. 34, no. 3, pp. 48-51, 2008.

[10] J. I. Hong, J. Zhang, D. Wang, W. Zhang, and X. Liu, "Flow coefficient of rectangular notch throttle orifice in spool valve," Journal of Lanzhou University of Technology, vol. 36, no. 3, pp. 47-50, 2010.

[11] T. Zhang, Y. Yang, and X. He, "Research on flow coefficient of water hydraulic throttles," Machine Tool \& Hydraulics, no. 2, pp. 120-122, 2002.

[12] D. Wu, Modeling and Experimental Evaluation of a LoadSensing and Pressure Compensated Hydraulic System, University of Saskatchewan, Saskatoon, Canada, 2003.

[13] D. Wu, R. Burton, and G. Schoenau, "An empirical discharge coefficient model for orifice flow," International Journal of Fluid Power, vol. 3, no. 3, pp. 13-19, 2002.

[14] C. Srikanth and C. Bhasker, "Flow analysis in valve with moving grids through CFD techniques," Advances in Engineering Software, vol. 40, no. 3, pp. 193-201, 2009.

[15] L. Pace, M. Ferro, F. Fraternale, M. D. Vedova, A. Caimano, and P. Maggiore, "Comparative analysis of a hydraulic servovalve," International Journal of Fluid Power, vol. 14, no. 2, pp. 53-62, 2013.

[16] W. Liu, T. Zhang, Y. Xu, and L. Bai, "Analysis on flow field in cone wake flow field," Journal of Tianjin University (Science and Technology), vol. 48, no. 10, pp. 929-936, 2015.

[17] H. Yu, T. Zhang, and W. Xu, "Influence of orifice chamfering on the flow field characteristic of multi-hole orifice flowmeter," Journal of Electronic Measurement and Instrumentation, vol. 29, no. 9, pp. 1356-1364, 2015.

[18] D. Gao, Numerical Simulation and Visualization Study on the Flow Field of Complicated Flow Channel in Hydraulic Techniques, Yanshan University, Qinghuangdao, China, 2011.

[19] J. Wang, Z. Wu, S. Cen, and X. Wang, "Flow field analysis and structural optimizationof hydraulic poppet valve based on 
ANSYS," Machine Tool \& Hydraulics, vol. 39, no. 13, pp. 128-130, 2011.

[20] X. Fu and X. Du, "Cavitation observation and flow-field analysis of high-speed flowinside throttling groove," Machine Tool \& Hydraulics, no. 7, pp. 29-31, 2006.

[21] C. Vallet, J. Ferrari, and J. F. Rit, "Single phase CFD inside a water safety valve," in Proceedings of The Asme2010 Pressure Vessels and Piping Division/K-Pvp Conference, pp. 335-342, American Society of Mechanical Engineers, Washington, DC, USA, July 2010. 


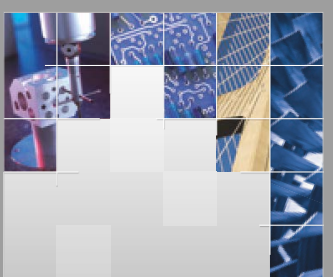

\section{Enfincering}
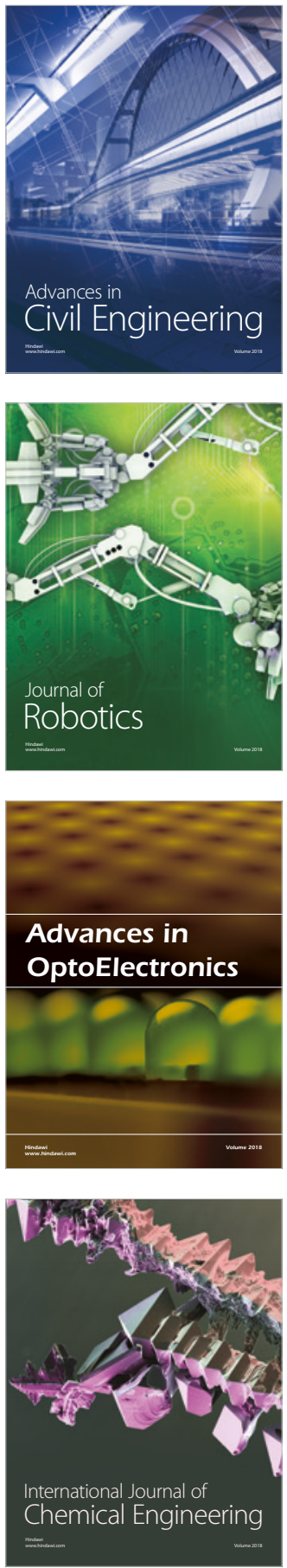

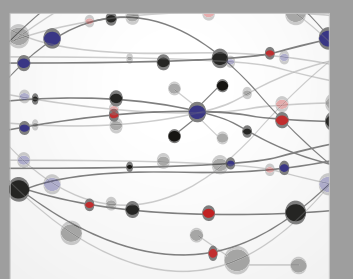

\section{Rotating \\ Machinery}

The Scientific World Journal

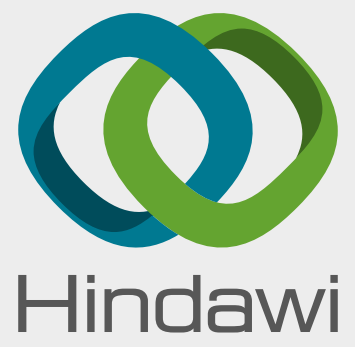

Submit your manuscripts at

www.hindawi.com
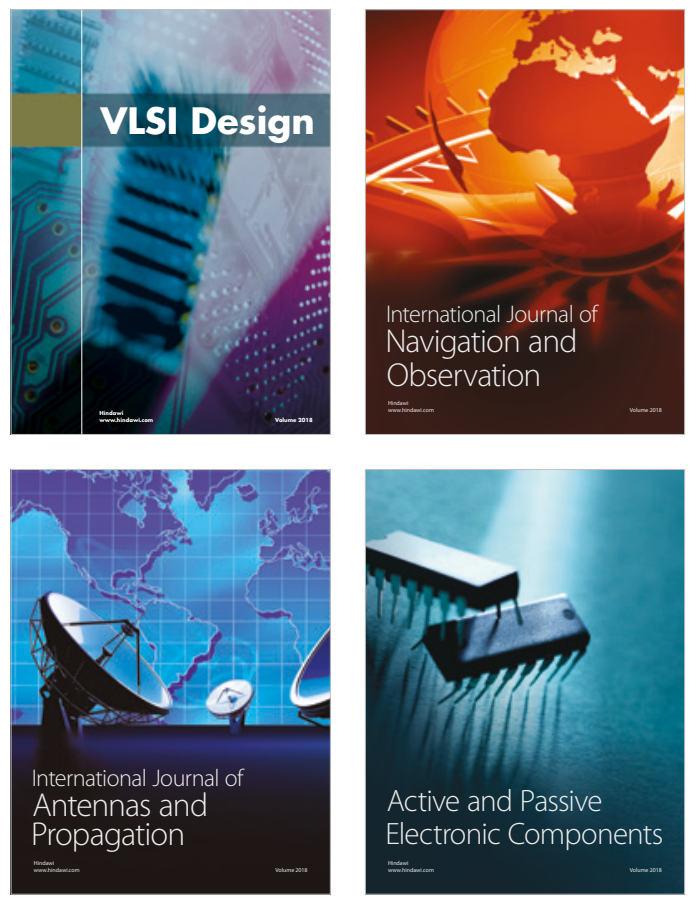
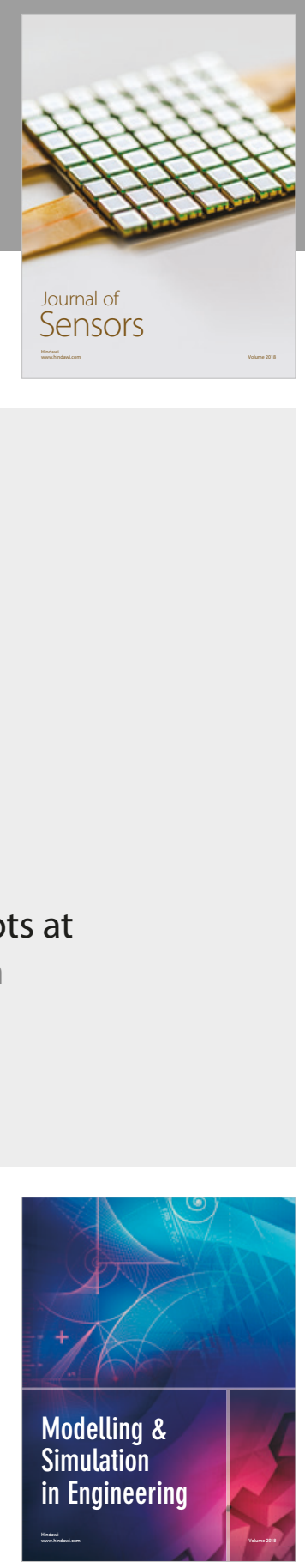

\section{Advances \\ Multimedia}
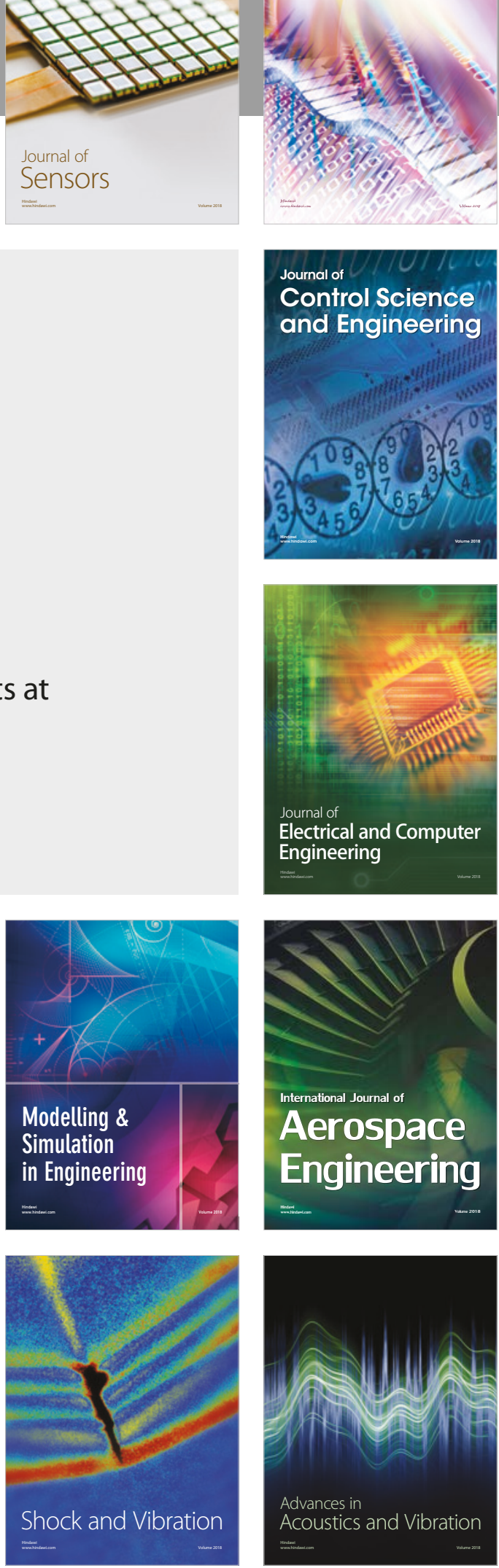